\title{
Glimmers of a Quantum KAM Theorem: Insights from Quantum Quenches in One-Dimensional Bose Gases
}

\author{
G. P. Brandino, ${ }^{1}$ J.-S. Caux, ${ }^{1}$ and R. M. Konik ${ }^{2, *}$ \\ ${ }^{1}$ Institute for Theoretical Physics, University of Amsterdam, \\ Science Park 904, 1090 GL Amsterdam, The Netherlands \\ ${ }^{2}$ CMPMS Department, Building 734, Brookhaven National Laboratory, Upton, New York 11973, USA \\ (Received 30 August 2014; revised manuscript received 26 July 2015; published 16 December 2015)
}

Real-time dynamics in a quantum many-body system are inherently complicated and hence difficult to predict. There are, however, a special set of systems where these dynamics are theoretically tractable: integrable models. Such models possess nontrivial conserved quantities beyond energy and momentum. These quantities are believed to control dynamics and thermalization in low-dimensional atomic gases as well as in quantum spin chains. But what happens when the special symmetries leading to the existence of the extra conserved quantities are broken? Is there any memory of the quantities if the breaking is weak? Here, in the presence of weak integrability breaking, we show that it is possible to construct residual quasiconserved quantities, thus providing a quantum analog to the KAM theorem and its attendant Nekhoreshev estimates. We demonstrate this construction explicitly in the context of quantum quenches in one-dimensional Bose gases and argue that these quasiconserved quantities can be probed experimentally.

DOI: 10.1103/PhysRevX.5.041043

\section{INTRODUCTION}

A milestone in the dynamics of classical many-body systems is the Kolmogorov-Arnold-Moser (KAM) theory [1]. Generically, classical many-body systems exhibit chaotic behavior - that is to say, giving the bodies of such systems slightly different initial positions and velocities results in the bodies following radically different trajectories. An exception to this rule is made for a special set of systems, termed integrable, which possess conserved quantities beyond energy and momentum. The existence of these conserved quantities promises the availability of a set of action-angle variables $\left\{p_{i}, q_{i}\right\}$ where the action variables, $p_{i}$, are constants of motion. In such variables, the Hamiltonian $H$ is solely a function of $\left\{p_{i}\right\}$, and the Hamiltonian equations of motion become particularly simple:

$$
\dot{q}_{i}=\frac{\partial H}{\partial p_{i}}, \quad \dot{p}_{i}=0 .
$$

Trajectories of bodies in integrable systems are not sensitive to initial conditions but instead lie on invariant tori in phase space described by frequencies $\left\{\omega_{i}\right\}$ parametrizing solutions to the equations of motion: $\dot{q}_{i}=\omega_{i}$. However,

rmk@bnl.gov

Published by the American Physical Society under the terms of the Creative Commons Attribution 3.0 License. Further distribution of this work must maintain attribution to the author(s) and the published article's title, journal citation, and DOI.
Subject Areas: Atomic and Molecular Physics,

Statistical Physics,

Strongly Correlated Materials integrable systems and their attendant simple behavior are comparatively rare. And so the question arises, what can one expect with a system that is merely close to being integrable. Is the motion of bodies in this system chaotic? Or is there some influence on the system's dynamics from being close to an integrable point? One answer to this question is given by the KAM theorem. It tells us that if we weakly perturb a classical integrable system, we do not immediately transit to completely chaotic dynamics, but rather see a smooth crossover. Specifically, the KAM theorem promises that a subset of the solutions $\left\{\omega_{i}\right\}$ survive under a sufficiently small perturbation, $\epsilon H_{\text {pert }}\left(p_{i}, q_{i}\right)$, provided their frequencies are sufficiently irrational.

What of quantum analogs to the KAM theorem? There is tremendous interest [2-29] in the role exotic conserved quantities play in the dynamics of lowdimensional systems. This interest $[4-11,19,20]$ arises in the context of one-dimensional (1D) Bose gases from the ability to manipulate isolated gases and observe their relaxation in closed surroundings, both when the gases are near integrable points [30-32] and when they are far away [33]. In the context of quantum spin chains [12-18], it comes about from the wish to understand related thermalization questions as well as whether integrable systems can sustain ballistic transport. It also appears in the burgeoning field of many-body localization [34,35] of disordered interacting systems and associated attempts to construct sequences of conserved charges in what one would traditionally consider a nonintegrable setting [36,37]. 
To understand crossover behavior arising from integrability breaking, both indirect measures such as level spacing statistics [38-41] and studies of systems in their quasiclassical limit using such tools as the semiclassical eigenfunction hypothesis [42-44] are often employed. Such behavior is often phrased in terms of prethermalization [45-50] or prerelaxation plateaus [51-53], where a system's observables, in relaxing from some nonequilibrium initial state, remain nearly constant over some finite time interval before decaying to their final equilibrium value. Such plateaus have been argued to be controlled by the remnants of the conserved quantities of the nearby integrable system $[48,49]$.

In this work, we go beyond this and show that, in finite systems, it is possible to construct an infinite sequence of nearly conserved local operators, $\left\{\mathcal{Q}_{i}\right\}_{i=1}^{\infty}$, in the presence of a perturbation that weakly breaks integrability,

$$
H=H_{\text {int }}+\epsilon \Phi_{\text {pert }} .
$$

We show that this near-conservation is good for all times. The $\mathcal{Q}_{i}$ are conserved in the sense that if we consider a (noneigen)state $|s\rangle$, with average energy per particle $E=\langle s|H| s\rangle / N$ less than some bound $\Lambda\left(N_{Q}\right)$, then

$$
\partial_{t}\left\langle s\left|\mathcal{Q}_{i}(t)\right| s\right\rangle<\delta\left(\epsilon, N_{Q}\right),
$$

for all times where $\delta\left(\epsilon, N_{Q}\right)$ can be made arbitrarily small. These conserved operators are constructed as finite linear combinations of length $N_{Q}$ involving the charges $\left\{\hat{Q}_{i}\right\}_{i}^{\infty}$ of the unperturbed Hamiltonian, $H_{\text {int }}$ :

$$
\mathcal{Q}_{i}\left(N_{Q}\right)=\sum_{j=1}^{N_{Q}} a_{i, j} \hat{Q}_{j+i N_{Q}} .
$$

The quality of this conservation can be controlled (i.e., $\Lambda$ can be made larger and $\delta$ smaller) by adjusting how many, $N_{Q}$, of the charges, $\hat{Q}_{i}$, appear in the linear combinations.

Our construction is akin not so much to the KAM theorem, but to what are known as Nekhoroshev estimates $[54,55]$ inasmuch as the charges $\mathcal{Q}$ we construct are nearly conserved on the entirety of the low-energy Hilbert space. While the KAM theorem promises that some subset of solutions of the equations of motion survive a perturbation and remain "close" to their integrable counterparts for all time, the Nekhoroshev estimates tell us that all solutions remain close to their integrable counterparts in the sense that

$$
\left|p_{i}(t)-p_{i}(0)\right|<P_{*} \epsilon^{1 /(2 N)},
$$

for exponentially long times:

$$
t<T_{*} e^{(a / \epsilon)^{1 /(2 N)}},
$$

where $P_{*}, T_{*}$, and $a$ are constants and $N$ is the number of degrees of freedom that the system has [54].

While general, we perform this construction in the context of quantum quenches in 1D Bose gases. This setting is particularly appropriate as it is the experimental study of quantum quenches in these gases [30] that has led to tremendous interest in the role of exotic conserved quantities in quantum dynamics. Quenches are, moreover, directly relevant to understanding these experiments. Because of the one-body potentials that trap the gases, they can be, at most, approximately integrable. Thus, the construction of a quantum version of the KAM theorem and its variants can only help yield insights into the dynamics of these gases in their experimental settings.

\section{QUANTUM QUENCH DYNAMICS IN 1D BOSE GASES}

To set the scene, we first describe the quantum quench in a 1D Bose gas as described by the Lieb-Liniger model [56]. The Lieb-Liniger model is believed to provide an excellent description of a 1D Bose gas [57]. In the absence of external (trapping) one-body terms, it is integrable with an infinite number of conserved operators, $\left\{\hat{Q}_{i}\right\}$. Its Hamiltonian, with the addition of a one-body potential $V(x)$, is given by

$H=-\frac{\hbar^{2}}{2 m} \sum_{j=1}^{N} \frac{\partial^{2}}{\partial x_{j}^{2}}+2 c \sum_{\langle i, j\rangle} \delta\left(x_{i}-x_{j}\right)+\sum_{i} V\left(x_{i}\right)$.

The type of quantum quench we study is found in preparing the gas on a ring of length $L$ in the ground state of a parabolic trap $[8,19,20]$, i.e., $V(x)=\frac{1}{2} m \omega^{2} x^{2}$, then at time $t=0$, releasing the gas from the parabolic trap into a one-body cosine potential, $V(x)=A \cos \left(2 \pi n_{\cos } x / L\right)$, and observing the subsequent dynamics of the gas. This quench protocol is illustrated in Fig. 1.

This form of the Hamiltonian, an integrable model together with an integrability-breaking perturbation, allows us to determine the ground and excited states of the model pre- and post-quench through a numerical renormalization group (NRG) designed precisely to attack such problems $[8,39,58,59]$, together with a set of routines known as ABACUS [60] that allow numerically exact computation of matrix elements of operators in the Lieb-Liniger model [61]. In turn, this gives us access to the post-quench dynamics of the gas. In particular, we employ a NRG able to study perturbations of integrable and conformal continuum field theories. This approach, as it is an extension of a methodology known as the truncated conformal spectrum approach $[62,63]$, has been primarily used to study perturbations of relativistic field theories [39,58,59], but it has recently been applied to the Lieb-Liniger model perturbed by a one-body potential [8], the problem at hand. The NRG uses the eigenstates of the Lieb-Liniger model as a 


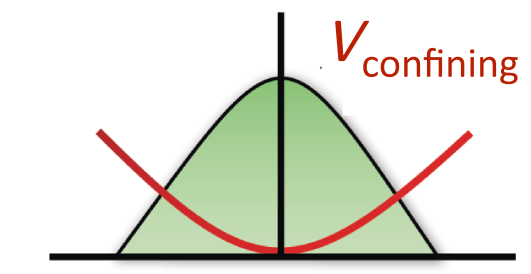

gas in a parabolic potential, $t<0$

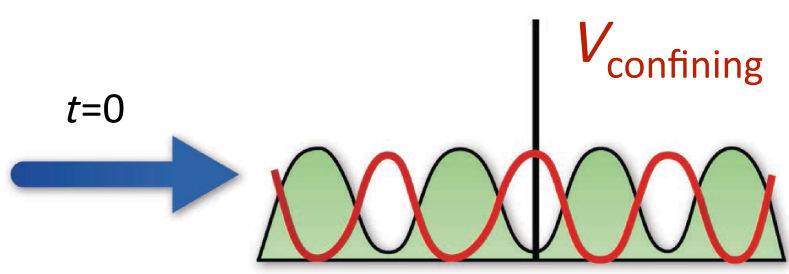

gas in a cosine potential, $t>0$

FIG. 1. Quench protocol: We prepare the one-dimensional Bose gas in its ground state in a harmonic trap. At time $t=0$, we release the gas into a cosine potential and track the subsequent dynamics. The shaded green regions are illustrations of the equilibrium density profiles of the gas in the presence of the confining potentials.

computational basis. Because this basis accounts for the interactions of the Bose gas particles with one another, this numerical method builds in the strong correlations present in the problem right at the start. We discuss details of this method in Appendix A 1.

In Fig. 2, we show the time evolution of the gas after the quench. At time $t=0$, we see the density profile of the gas

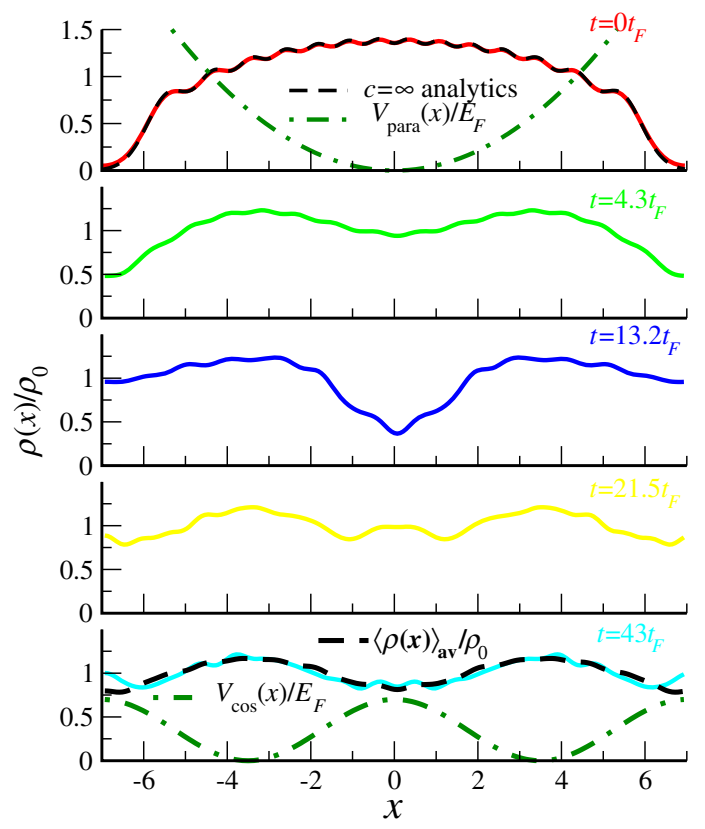

FIG. 2. The density profile of the gas at selected times postquench as computed with the NRG. Here, this time dependence is computed after releasing an $N=L=14, c=7200$ gas prepared in a parabolic potential with $m \omega_{0}^{2} L^{2} / 2 E_{F}=10.36$ [shown with a green dashed line in the $t=0 t_{F}$ frame, $t_{F}=1 / E_{F}$, $E_{F}=k_{F}^{2} /(2 m)$, and $\left.k_{F}=\pi(N-1) / L\right]$ into a cosine potential $V_{\cos }(x) / E_{F}=0.35(\cos [(4 \pi / L) x]+1)$ (plotted with a dashed line in the $t=43 t_{F}$ frame). In the $t=0$ frame, we show the density profile as computed analytically in the hard-core limit (see Appendix A 2). Using the NRG, we can run the time evolution as far out as $t=85 t_{F}$ before dephasing exceeds $1 \%$. We see, however, by $t=43 t_{F}$ the gas's density profile has already come close to its long time average (black dashed line in the final panel). in the ground state of the parabolic potential. After quenching the potential to a cosine, the gas moves away from the center, oscillates a number of times before settling into the minima of the cosine. This occurs at times of the order of $t=50 t_{F}$-we are able to run the simulation out to times of $t=80 t_{F}$ [here, $t_{F}=1 / E_{F}$, where $E_{F}=k_{F}^{2} /(2 m)$ and $\left.k_{F}=\pi(N-1) / L\right]$.

While we are able to compute the dynamics of such observables as the density and the momentum distribution function, the key to the work in this paper is our ability to compute the dynamics of the (formerly) conserved LiebLiniger charges, $\hat{Q}_{i}$. Our numerical approach makes this extremely simple because of our use of the eigenstates of the integrable Lieb-Liniger model as a basis. Each LiebLiniger state of an $N$-particle gas $|\psi\rangle_{L L}$ is characterized by $N$-rapidities, $\lambda_{i}, i=1, \ldots, N$, which should be thought of as, more or less, the momenta of the gas's particles. These rapidities determine the action of the conserved operators on the Lieb-Liniger states. For example, both the energy, $E=\hat{Q}_{2}$, and momentum, $P=\hat{Q}_{1}$, operators act on $|\psi\rangle_{L L}$ via (taking $m=1 / 2$ )

$E|\psi\rangle_{L L}=\sum_{i=1}^{N} \frac{\lambda_{i}^{2}}{2 m}|\psi\rangle_{L L} ; \quad P|\psi\rangle_{L L}=\sum_{i=1}^{N} \lambda_{i}|\psi\rangle_{L L}$

The action of all of the higher nontrivial charges, $\hat{Q}_{n}$, $n=3,4,5, \ldots$, in the Lieb-Liniger model are simply higher power sums of the same rapidities:

$$
\hat{Q}_{n}|\psi\rangle_{L L}=\sum_{i=1}^{N} \lambda_{i}^{n}|\psi\rangle_{L L}
$$

While the actual expression of the charges in terms of the Bose field operators is complicated and unwieldy [64], the action of the charges on the Lieb-Liniger eigenstates turns out to be extremely simple. This will be crucial in facilitating our construction of effective $\mathcal{Q}$ 's. 


\section{CONSTRUCTION OF CONSERVED QUANTITIES IN THE BOSE GAS POST-QUENCH}

We now turn to the core of the paper. We have shown, in the previous section, that we can describe the temporal dynamics of various quantities post-quench. In that section, we specifically considered the density profile of the gas after release into the cosine potential. We now consider the time evolution of the Lieb-Liniger charges. They are, of course, not conserved, and so their evolution will be nontrivial. We however show that one can construct linear combinations of the Lieb-Liniger charges whose expectation values are nearly time invariant under evolution by the post-quench Hamiltonian. The quality of this time invariance can be controlled by allowing more charges in the linear combination. Moreover, we show that these linear combinations of charge are not merely time invariant with respect to the particular initial condition created in the quench protocol, but also as operators acting on the low-energy Hilbert space.

We begin by first considering the time evolution of the individual Lieb-Liniger charges themselves. We plot this evolution for the first four Lieb-Liniger charges in Fig. 3 for a gas with $N=L=8$ and $c=10$. In plotting the time evolution, we have normalized each charge to its mean value post-quench so that all of the charges fluctuate about 1 . The mean value of the unnormalized $n$th charge is given by

$$
\left\langle\hat{Q}_{n}\right\rangle_{\mathrm{av}}=\frac{1}{T} \int_{0}^{T}\left\langle\hat{Q}_{n}(t)\right\rangle
$$

where $T$ is the time out to which we can track the evolution. This mean value grows rapidly with $n$. This happens because the charge's action on a Lieb-Liniger eigenstate $|s\rangle=$ $\left|\lambda_{1}, \ldots, \lambda_{N}\right\rangle$ is a power sum of the rapidities $\left\{\lambda_{i}\right\}_{i=1}^{N}$, i.e., $\left\langle s\left|\hat{Q}_{n}\right| s\right\rangle=\sum_{i} \lambda_{i}^{n}$. We see from Fig. 3 that, even after normalization, the size of the oscillations increases with $n$.

We now consider linear combinations of the LiebLiniger charges of the form

$\mathcal{Q}\left(N_{Q}\right)=a_{0} \mathrm{I}+\sum_{i=1}^{N_{Q}} \frac{a_{i}}{\left\langle\hat{Q}_{2 i}\right\rangle_{\mathrm{av}}} \hat{Q}_{2 i} ; \quad 1=\sum_{i=1}^{N_{Q}}\left|a_{i}\right|^{2}$,

where we choose the constant $a_{0}$ such that the mean value of $\mathcal{Q}\left(N_{Q}\right)$ is 0 and the remaining constants $a_{i}$ [65] such that the fluctuations in $\mathcal{Q}\left(N_{Q}\right)$ are minimized.

We plot the time evolution for a $c=10$ gas of these effective charges in panel (b) of Fig. 3 for three different values of $N_{Q}$, the number of charges in the linear combination. In panel (c), we plot the fluctuations of this charge as a function of $N_{Q}$. We see that these fluctuations drop exponentially with $N_{Q}$. (On the basis of an error analysis in our numerics, we would put a numerically induced floor of $10^{-6}$ to $10^{-7}$ on the fluctuations in $\mathcal{Q}$ - see end of Appendix A 1 a.) In the bottom part of panel (c), we do the same for a quench involving a $c=1$ gas. In order to be sure that we are not simply reconstructing the post-quench Hamiltonian as some linear combination of the Lieb-Liniger charges, in both cases $(c=10,1)$, we demonstrate that we can construct simultaneous multiple effective charges. In panel (c), we show that
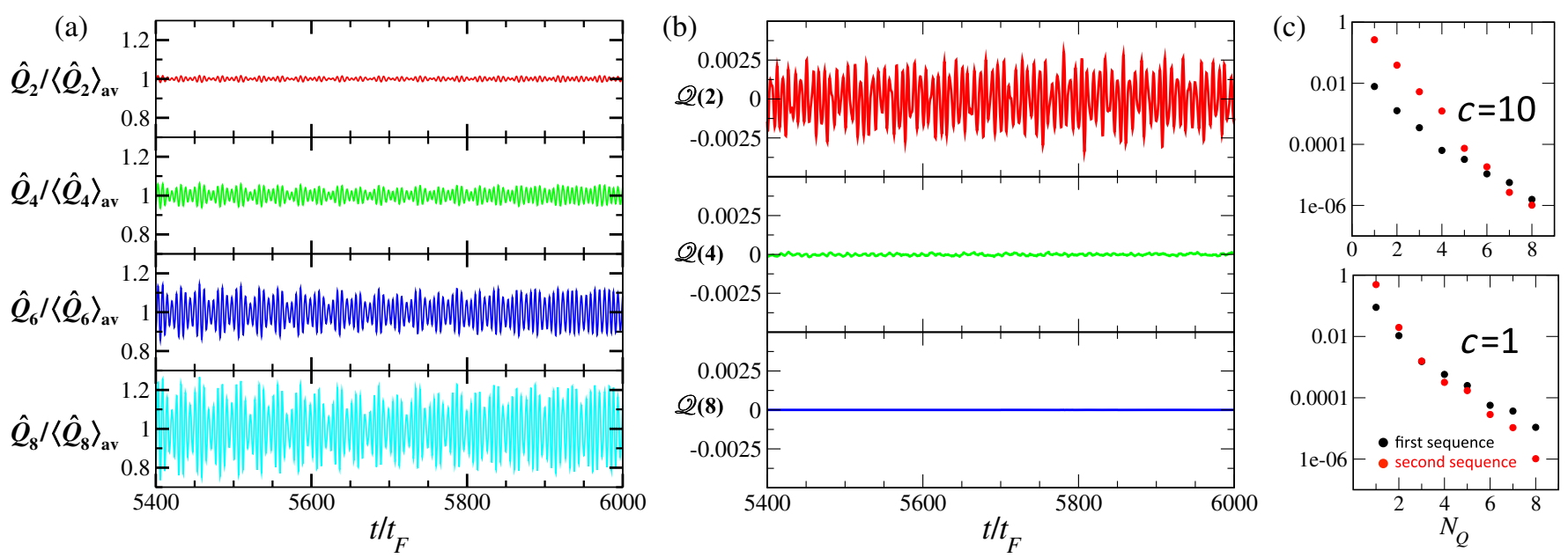

FIG. 3. (a) The post-quench time evolution of the Lieb-Liniger charges normalized by their mean value as described in the text, $\hat{Q}_{i} /\left\langle\hat{Q}_{i}\right\rangle_{\mathrm{av}}$. Here, the time dependence is computed after releasing an $N=L=8, c=10$ gas prepared in a parabolic potential of strength $m \omega_{0}^{2} L^{2} / 2 E_{F}=3.24$ into a cosine potential $\cos [(4 \pi / L) x]$. We show this behavior at late times (for details of how long we can run the simulation, see Appendix A 1). (b) The post-quench time evolution of a sequence of effective charges with the mean subtracted off, $\mathcal{Q}\left(N_{Q}\right)=\sum_{m=1}^{N_{Q}} a_{2 m} \hat{Q}_{2 m}$, for $N_{Q}=2,4$, and 8. (c) Top panel: The standard deviation of the fluctuations of two sequences of effective charges $\mathcal{Q}$. We build the first sequence (in black) using linear combinations of the charges $\left\{\hat{Q}_{2 m}\right\}_{m=1}^{m=8}$, while the second sequence (in red) is formed with the next eight Lieb-Liniger charges, i.e., $\left\{\hat{Q}_{2 m}\right\}_{m=9}^{m=16}$. Bottom panel: We show the fluctuations of the two effective charges built following the quench of a $c=1$ gas prepared in a parabolic trap of strength $m \omega_{0}^{2} L^{2} / 2 E_{F}=0.13$ and released into the same cosine potential, $\cos [(4 \pi / L) x]$. 


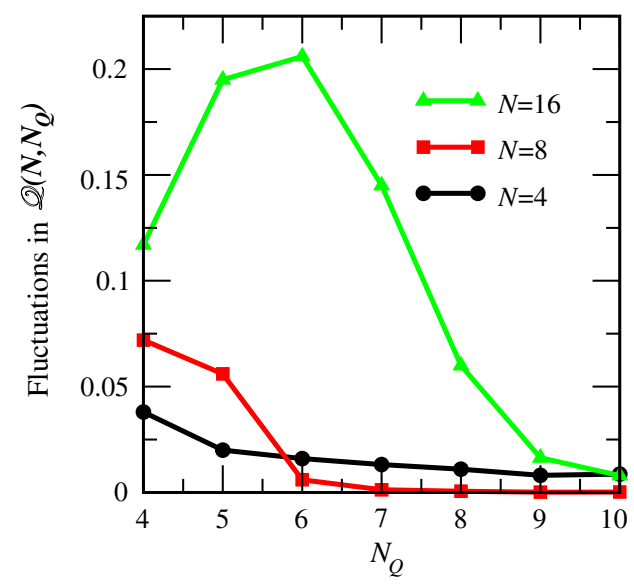

FIG. 4. We plot the fluctuations in time for $\mathcal{Q}$ as a function of $N_{Q}$ for $N=L=4,8$, and 16 for a quench in a $c=10$ gas from a parabolic potential of strength $m \omega_{0}^{2} L^{2} / 2 E_{F}=2.33$ to a cosine of amplitude $V_{\cos }(x)=0.26 E_{F} \cos (2 \pi x / L)$. We do so using the charges constructed at $c=\infty$, as discussed in Appendix B, as a partial demonstration that such charges work well at finite $c$.

the fluctuations of a second effective charge built as a linear combination of charges drawn from $\left\{\hat{Q}_{2 n}\right\}_{n=9}^{16}$ also die off exponentially.

This exponential dependence in $N_{Q}$ is possible to understand at large $c$. To do so, we write the initial condition of the gas in terms of post-quench cosine eigenstates: $\left|\psi_{\mathrm{GS}}\right\rangle=$ $\sum_{\alpha} c_{\alpha}\left|\psi_{\alpha, \text { cos }}\right\rangle$. With the initial condition as above, the time dependence of the charge takes the form

$$
\begin{aligned}
\mathcal{Q}(t) & =\sum_{\alpha \beta} c_{\alpha}^{*} c_{\beta}\left\langle\psi_{\alpha, \cos }|\mathcal{Q}(t)| \psi_{\beta, \cos }\right\rangle \\
& =\sum_{\alpha \beta} c_{\alpha}^{*} c_{\beta} e^{-i\left(E_{\beta}-E_{\alpha}\right) t}\left\langle\psi_{\alpha, \cos }|\mathcal{Q}| \psi_{\beta, \cos }\right\rangle .
\end{aligned}
$$

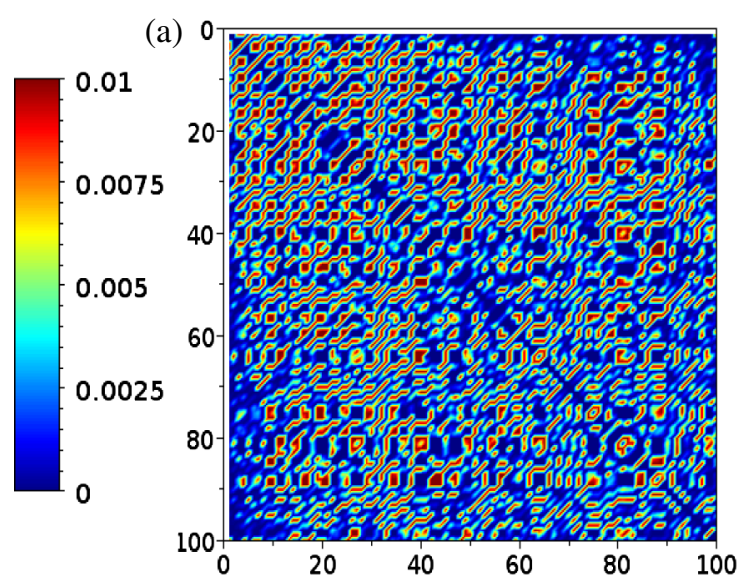

(b)

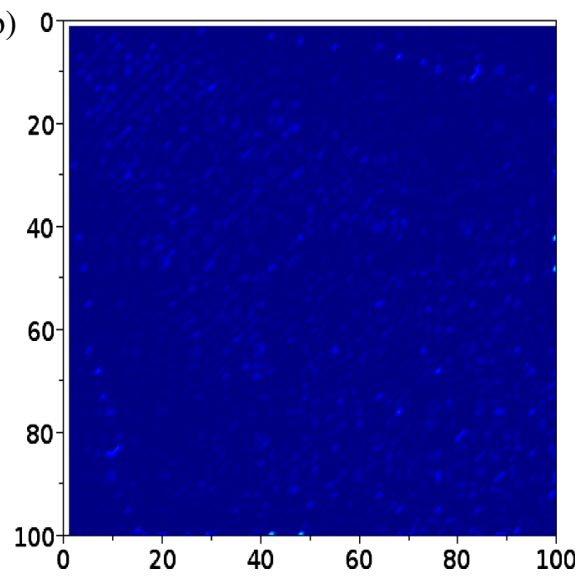

We demonstrate in Appendix B 1 that each Lieb-Liniger charge forming $\mathcal{Q}$ zeros out a shell of matrix elements $\left\langle\psi_{\alpha, \cos }|\mathcal{Q}| \psi_{\beta, \cos }\right\rangle, \alpha \neq \beta$, in the above sum. As $N_{Q}$ increases, more and more of these matrix elements are zeroed out. For relatively weak cosine potentials, the total weight $W_{\text {elim }}$ of the $\left|c_{\beta} c_{\alpha}\right|^{2}$ 's whose matrix elements are zeroed out is

$$
W_{\mathrm{elim}} \approx 1-2 \frac{e^{-\Lambda\left(N_{Q}\right)^{2}}}{\sqrt{\pi}} \sum_{n=0}^{N-1} \frac{2^{n} \Lambda\left(N_{Q}\right)^{2 n-1}}{n !},
$$

with $\Lambda\left(N_{Q}\right)=\left(2 \pi\left(N_{Q}-2\right) /\left(L \sqrt{m \omega_{0}}\right)\right.$. We then see the weight that is not zeroed out, and thus can contribute to $\mathcal{Q}$ 's temporal fluctuations, goes as $e^{-\left(\Lambda\left(N_{Q}\right) / m \omega_{0}\right)^{2}}$. We see from this that it becomes harder to construct quasistationary $\mathcal{Q}$ 's as the system size $L$ is increased. This is confirmed in Fig. 4, where we compare $\mathcal{Q}$ 's constructed at different $N=L$. We see that the point where the fluctuations become exponentially small goes as $N_{Q}=L$.

For large-amplitude $A$ cosine potentials, the temporal fluctuations die off much more slowly with $N_{Q}$ :

$$
W_{\text {elim }} \sim\left(\frac{N_{Q}}{N_{A}}\right)^{N}, \quad N_{A}=\frac{\sqrt{2 m A} L}{2 \pi} .
$$

In this latter case, the number of nonzero matrix elements of $\mathcal{Q}(t)$ proliferate, making a construction where the effective charge is nearly time invariant much more difficult.

So far, we have only demonstrated that we can construct charges $\mathcal{Q}$ as linear combinations of the original LiebLiniger charges $\hat{Q}_{n}$ whose time fluctuations can be made arbitrarily small when we start the system at a specific initial condition, $\left|\psi_{\mathrm{GS} \text {,para }}\right\rangle$. However, we now demonstrate that these charges are quasiconserved not just relative to a

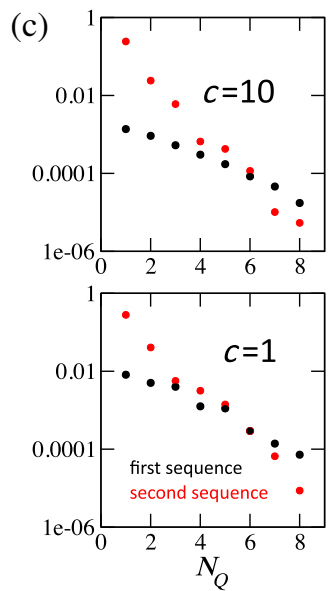

FIG. 5. (a) We plot the intensity of the off-diagonal matrix elements of $\hat{Q}_{2} /\left\langle\hat{Q}_{2}\right\rangle_{\mathrm{av}}$, comparing it to (b) the off diagonal m.e.'s of $\mathcal{Q}(8)$ for the quench of the $c=1$ gas discussed in Fig. 3(c). (c) We plot the average size of the off-diagonal matrix elements of two sequences of effective charges $\mathcal{Q}\left(N_{Q}\right)$; in black is the sequence constructed from $\hat{Q}_{2 m}, m=1, \ldots, 8$, while in red is the sequence constructed from $\hat{Q}_{2 m}$, $m=9, \ldots, 16$. We show this for both the $c=1$ [same quench as in (a) and (b)] and the $c=10$ case [same quench as described in Figs. 3(a)-(c)]. 
specific initial state but as operators, at least when projected onto the low-energy post-quench Hilbert space.

To do so, we compute the off-diagonal matrix elements in Fig. 5 of one of the two $\mathcal{Q}$ 's we have constructed (the one constructed with Lieb-Liniger charges, $\left.\hat{Q}_{2}, \ldots, \hat{Q}_{16}\right)$ relative to the basis of the low-lying energy eigenstates of the post-quench Hamiltonian. These matrix elements are plotted in Fig. 5. In the rightmost panel, we display the offdiagonal matrix elements of $\hat{Q}_{2}$ (normalized as described previously) to set the scale of how large these matrix elements are for the individual Lieb-Liniger charges. In the middle panel, we then plot the matrix elements of $\mathcal{Q}(8)$. We see that most of the previous nonzero matrix elements of $\hat{Q}_{2}$ are now dramatically reduced. We quantify this disappearance in panel (c) of Fig. 5. There, we present the average magnitude of the off-diagonal matrix elements as a function of $N_{Q}$. We present data for both effective charges considered in Fig. 3 for both values of $c=1,10$. We see, in all cases, that the size of these matrix elements drops exponentially in $N_{Q}$. Roughly speaking, if the average energy per particle of two distinct states, $|s\rangle,\left|s^{\prime}\right\rangle$, is less than $\Lambda\left(N_{Q}\right)$, then $\left\langle s|\mathcal{Q}| s^{\prime}\right\rangle$ will be exponentially small. We conclude that the $\mathcal{Q}$ 's are then nearly conserved as operators. This conclusion is supported by an analytic construction of the $\mathcal{Q}$ 's that we present in Appendix B.

\section{DISCUSSION}

In this paper, we have found the construction of quasiconserved operators as linear combinations of the Lieb-Liniger conserved charges. In this construction, the linear combinations are chosen to minimize the temporal fluctuations of the charge upon quenching the gas from a one-body parabolic potential to a cosine potential. Even though this minimization is being done for a particular quench protocol, the conservation of the charge occurs at the operator level. Specifically, off-diagonal matrix elements of the charges are small. We demonstrated that both post-quench temporal fluctuations and the off-diagonal matrix elements can be made exponentially small in the number of charges, $N_{Q}$, in the linear combination. We have supported this construction by demonstrating an equivalent analytic construction of these charges (Appendix B).

In this analytic construction of effective charges, we demonstrate why certain linear combinations of the original Lieb-Liniger charges act as effective conserved quantities at low energies. This construction works by finding linear combinations that zero out off-diagonal matrix elements at a given order in the effective charge $\mathcal{Q}(t)$ written as a power series in time $t$. We show, in particular, that a matrix element zeroed out at a given order in $t$ remains zero to a much higher order. This provides an explanation as to why our construction appears so robust. We emphasize here that this does not use the $c=\infty$ integrability of the Lieb-Liniger model in the cosine potential. (At $c=\infty$ the Lieb-Liniger model plus arbitrary one-body potential is generically integrable because the model maps onto free fermions.) However, to reassure the reader that our $c=\infty$ construction is not accidentally constructing these occupation numbers, we demonstrate that the charges we analytically construct at $c=\infty$ work at finite $c$ as well. In Fig. 6 we plot the temporal fluctuations of these analytically constructed charges as a function of $N_{Q}$ for the $c=1$ and $c=10$ quenches described in Fig. 3. While we see that the temporal fluctuations of these analytical $c=\infty$ charges are larger than those numerically constructed at a given $c$ [compare Fig. 3(c)], we nonetheless see that the fluctuations in the $c=\infty$ charges die off exponentially with $N_{Q}$. A similar conclusion can be seen in our study of the temporal fluctuations of $\mathcal{Q}$ as a function of $N$ and $N_{Q}$ in Fig. 4, where we have used the $c=\infty$ effective charges-although here, for the $N=4$ data, one can see that the fluctuations for the analytic $\mathcal{Q}$ have a comparatively large floor. Altogether, this gives us confidence that our $c=\infty$ construction is accurately capturing the essence of the numerical construction of $\mathcal{Q}$ at finite $c$.

In fact, we are able to extend the analytic computation described in Appendix B to the finite $c$ case. The primary difference between the construction of $\mathcal{Q}$ at $c=\infty$ and $c$ finite is the need to take into account that the density operator can connect states differing by more than one particle-hole pair. However, these higher particle-hole processes are suppressed in powers of $1 / c$, with $c$ the interaction strength. This means that we have a control parameter in our finite $c$ analytic computation of $\mathcal{Q}$ where if we ignore processes involving $n$-particle-hole pairs, the error we make is only $c^{-n}$. This in part explains why our $c=\infty$ construction of $\mathcal{Q}$ is still conserved at $c=10$. However, it is somewhat surprising that our $c=\infty$ construction of $\mathcal{Q}$ works as well as it does (as evidenced in

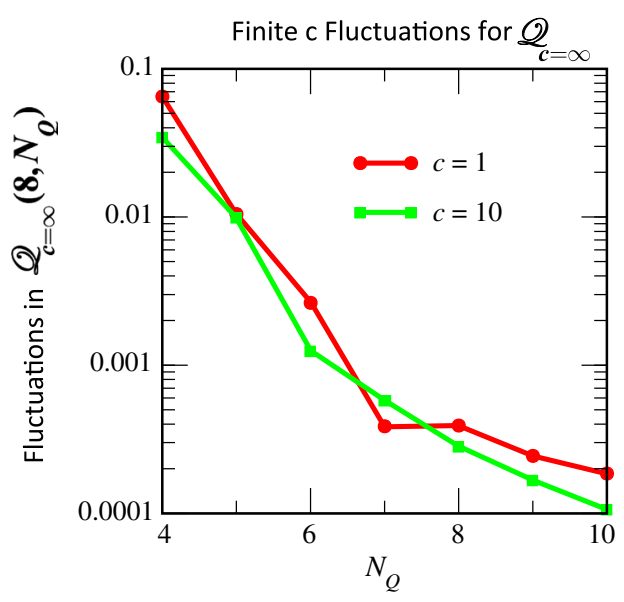

FIG. 6. We demonstrate that the effective charges constructed analytically at $c=\infty$, as described in detail in Appendix B, have suppressed temporal fluctuations for quenches with finite $c=1,10$. 
Fig. 6) for $c=1$. This suggests that higher particle-hole processes, at least for quenches whose dynamics are restricted to the low-energy post-quench Hilbert space, are unimportant.

In the Introduction to this paper, we have billed these constructions as being quantum equivalents to the classical KAM theorem and its counterparts such as the Nekhoreshev estimates. There are some similarities in the consequences of our constructions as well as some dissimilarities. Nekhoreshev estimates tell us that the values of the classical action variables in the face of a small nonintegrable perturbation change only very slowly in time, as controlled by both the size of the perturbation and the number of degrees of freedom [see Eqs. (5) and (6)].

For the quantum case, we see something analogous but with certain differences. These differences arise both because we are forming linear combinations of the originally conserved charges and because of how, in our construction, we segregate portions of the quantum phase (Hilbert) space. Nekhoreshev estimates apply to the entire phase space of the weakly perturbed model [i.e., Eq. (5) is good for any $\left.p_{i}(t=0)\right]$. In contrast, in our constructions, the approximate time invariance of the charge is restricted to a portion of the low-energy Hilbert space as marked by the integer $N_{\max }$ (this low-energy Hilbert space is defined by states where none of the particles in the state have momenta greater than $\left.k_{\max }=2 \pi N_{\max } / L\right)$. While we can make $N_{\max }$ as large as we want (provided we are willing to make $N_{Q}$ correspondingly large), we cannot take it to be infinite.

Another difference between the two constructions is the role played by the strength of the integrability-breaking perturbation. Here the Nekhoreshev estimates provide a bound on the temporal variation of the original action variables. This classical bound goes as $A^{1 /(2 N)}$ where $A$ is the perturbation's strength and $N$ is the number of degrees of freedom in the system. In contrast, we construct effective charges, $\mathcal{Q}$, whose temporal variation is controlled not only by $A$, but also by $N_{Q}$ the number of Lieb-Liniger charges forming $\mathcal{Q}$. To be sure, if $A$ is large, $N_{Q}$ will need to be correspondingly larger in order to produce the same minimum of temporal variation [see Eq. (14)].

In constructing these charges, the nature of the potential is important here. Our potential mixes the momenta of different (unperturbed) eigenstates solely through the wave vector of the cosine potential. This process is then considerably different than the integrability breaking considered in Refs. [66,67], where they considered integrability that respected no selection rules and correspondingly saw an extremely rapid crossover from quantum integrable to quantum chaoticity. However, this does not mean our construction of $\mathcal{Q}$ does not work if the potential induces nontrivial mixing between wave vectors. To this end, we considered preparing the system as normal in the ground state of a parabolic potential, but instead of releasing the gas into a cosine potential, we released it into a weaker parabola. In

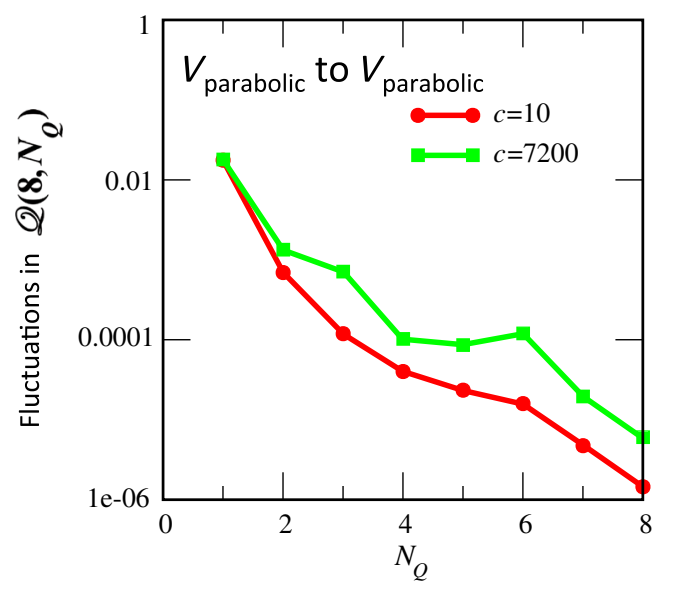

FIG. 7. We show that the fluctuations in the effective charges $\mathcal{Q}$ constructed from a quench from a stronger to a weaker parabolic potential, like their parabola to cosine counterparts, die out rapidly with $N_{Q}$. We consider two quenches of this type, one with the gas at $c=7200$ and one with $c=10$. For the $c=7200$ case, we quench from a parabolic potential with strength, $\omega_{0 \text {,init }}$ given by $m \omega_{0 \text {,init }}^{2} L^{2} / 2 E_{F}=6.48$ into a parabolic potential with strength $\omega_{0, \text { fin }}$ given by $m \omega_{0, \text { fin }}^{2} L^{2} / 2 E_{F}=2.11$. And for the $c=10$ case, we quench from a parabola described by $m \omega_{0, \text { init }}^{2} L^{2} / 2 E_{F}=3.24$ into one given by $m \omega_{0, \text { fin }}^{2} L^{2} / 2 E_{F}=1.06$.

Fig. 7, we show the fluctuations in $\mathcal{Q}\left(N_{Q}\right)$ as a function of the number $N_{Q}$ of Lieb-Liniger charges used to construct $\mathcal{Q}$. As with the release into the cosine potential, we are able to construct a sequence of $\mathcal{Q}\left(N_{Q}\right)$ whose temporal fluctuations die off rapidly with increasing $N_{Q}$. And although we do not show it, the off-diagonal matrix elements of these charges fall off as rapidly as their cosine counterparts in Fig. 5.

\section{EXPERIMENTAL CONSEQUENCES}

Having constructed these charges, we can ask what consequences follow from their existence. That they take nonzero values on the eigenstates means that the long time dynamics of the gas post-quench is going to be constrained. In this light, we have one way to understand the "quantum Newton's cradle" experiment presented in Ref. [30]. In the Introduction, we argued that the post-quench dynamics of a gas were very slow to achieve equilibration and that this slowness was indicative of the underlying integrability of the Lieb-Liniger model. However, strictly speaking, the gas in this experiment was not integrable. The gas was confined in a one-body parabolic potential, a potential that breaks integrability [68]. Our construction of effective quasiconserved charges in the presence of an integrability-breaking one-body potential thus provides a means to understand the slow thermalization of the gas post-quench in this experiment despite the presence of integrability breaking. More generally, our construction helps explain the findings of Refs. $[69,70]$, where weak integrability breaking does not lead to immediate thermalization in finite systems. 
In constructing these operators, it should be stressed that the operators we construct are local (in the sense that they are spatial integrals over operators that are defined at a single point in space). This follows as the effective charges $\mathcal{Q}$ are constructed as linear combinations of the Lieb-Liniger charges, which are all local quantities. Thus, we are not constructing, in effect, projection operators corresponding to eigenstates of the post-quench gas. Such projection operators are necessarily always present in a model regardless of its integrability. To demonstrate this we plot in Fig. 8 the diagonal matrix elements of the charge, $\mathcal{Q}(8)$, from the $c=1$ quench of Fig. 3. We see that these matrix elements are all non-vanishing and several orders of magnitude larger than $\mathcal{Q}(8)$ 's off-diagonal elements (see Fig. 5(c)).

If the nearly conserved quantities are governing the long time dynamics of 1D Bose gases as in Ref. [30], a second question that must be asked is whether this influence is merely confined to a prethermalization plateau or whether it influences the dynamics of the gas at all times. There are at least two constructions [48,49] of quasiconserved quantities that are thought to govern prethermalization plateaus. Our construction is fundamentally different inasmuch as the quasiconserved operators are quasiconserved all times. This fact, in particular, implies that a modified form of Mazur's inequality [71] holds. This inequality relates the long time average of a correlation function $\lim _{t \rightarrow \infty}\langle\mathcal{O}(t) \mathcal{O}(0)\rangle$ with the projection $\langle\mathcal{O} Q\rangle$ of the operators $\mathcal{O}$ onto conserved charges $Q$. This inequality continues to hold with quasiconserved charges $\mathcal{Q}$ but with the addition of an error term that is proportional to the size of $\mathcal{Q}$ 's off-diagonal matrix elements (which, in our construction, can be made arbitrarily small), something immediately clear from the proof of Mazur's inequality found in Ref. [72]. This implies that $\mathcal{Q}$ will control the long time limit of a host of experimental observables in systems with weak integrability breaking. We consider this further in the next subsection.

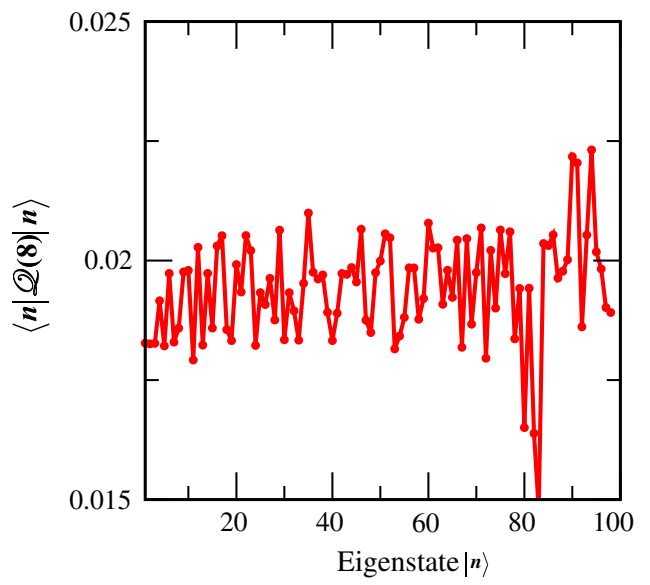

FIG. 8. We plot the values of the diagonal matrix elements of $\mathcal{Q}(8)$ in the post-quench eigenbasis as derived for the $c=1$ quench discussed previously in Figs. 3.

\section{A. $\mathcal{Q}$ and Mazur's inequality}

To understand Mazur's inequality [73] in the context of our effective charges, we adapt the argument presented in Ref. [72], establishing this inequality in the context of thermal correlation functions. To this end, we consider the following connected correlation function:

$$
\begin{aligned}
\chi_{k}= & \lim _{T \rightarrow \infty} \frac{1}{\left\langle M_{k}\right\rangle_{\mathrm{DE}}}\left[\frac{1}{T^{2}} \int_{0}^{T} d t d t_{0}\right. \\
& \left.\times\left(\left\langle M_{k}\left(t+t_{0}\right) M_{k}\left(t_{0}\right)\right\rangle-\left\langle M_{k}\right\rangle_{\mathrm{DE}}^{2}\right)\right]^{1 / 2} ; \\
\left\langle M_{k}\right\rangle_{\mathrm{DE}}= & \lim _{T \rightarrow \infty} \frac{1}{T} \int_{0}^{T} d t\left\langle M_{k}(t)\right\rangle .
\end{aligned}
$$

For the case at hand, the most relevant operator, $M_{k}$, to consider will be either the $k$-th Fourier component of the momentum distribution function (MDF) operator, i.e.,

$$
M_{k}(t)=\psi_{k}^{\dagger} \psi_{k},
$$

or the density operator

$$
M_{k}(t)=\frac{1}{L} \sum_{q} \psi_{k+q}^{\dagger} \psi_{k},
$$

where $\psi_{k}^{\dagger}$ is the $k$-th Fourier component of the Bose field. Here, we are averaging over both $t$ and $t_{0}$ in order to remove any dependence on the waiting time $t_{0}$. We have defined $\chi_{k}$ so that correlations are measured in units of $M_{k}$ computed in the long time limit, i.e., in the diagonal ensemble. We evaluate these correlation functions $\langle\cdots\rangle$ with respect to the initial condition of the gas in the ground state of a parabolic trap, $|i\rangle=\left|\psi_{\mathrm{GS} \text {,para }}\right\rangle \cdot \chi_{k}$ is nonzero only if there are correlations present in $M_{k}$ that survive the $t \rightarrow \infty$ limit, i.e.,

$$
\begin{aligned}
& \lim _{t \rightarrow \infty}\left\langle i\left|M_{k}\left(t+t_{0}\right) M_{k}\left(t_{0}\right)\right| i\right\rangle \\
& \quad \neq \lim _{t \rightarrow \infty}\left\langle i\left|M_{k}\left(t+t_{0}\right)\right| i\right\rangle\left\langle i\left|M_{k}\left(t_{0}\right)\right| i\right\rangle .
\end{aligned}
$$

The presence of similar long time correlations is precisely what guarantees a finite Drude weight in transport in integrable systems [12].

We demonstrate in Appendix $\mathrm{C}$ that a lower bound can be put on $\chi_{k}$ involving our effective charge $\mathcal{Q}$ of the form

$$
\chi_{k} \geq \frac{\left\langle i\left|M_{k} \mathcal{Q}_{\text {diag }}\right| i\right\rangle^{2}}{\left\langle i\left|\mathcal{Q}_{\text {diag }}^{2}\right| i\right\rangle},
$$

where $\mathcal{Q}_{\text {diag }}$ is the diagonal part of the effective charge $\mathcal{Q}$. If our initial condition state $|i\rangle=\mid \psi_{\mathrm{GS}, \text { para }}$ is confined to the low-energy Hilbert space where $\mathcal{Q}_{\text {diag }}$ and $\mathcal{Q}$ differ by off-diagonal matrix elements of size $\mathcal{O}(\delta)$, we can rewrite this inequality as 


$$
\chi_{k} \geq \frac{\left\langle i\left|M_{k} \mathcal{Q}\right| i\right\rangle^{2}}{\left\langle i\left|\mathcal{Q}^{2}\right| i\right\rangle}+\mathcal{O}(\delta)
$$

as claimed at the end of the last subsection.

We now show that this lower bound arising from $\mathcal{Q}$ on $\chi_{k}$ is, in fact, finite. In Fig. 9, we plot this lower bound for both correlations involving the MDF operator and the density operator. We study this lower bound at three different system sizes and three different wave vectors. We see that, in all cases, this lower bound is appreciable. For the MDF, the lower bound on $\chi_{k}$ is such that the correlations in this quantity are at least roughly at the $10 \%$ level. To determine whether this is significant, we compute a similar lower bound for a quench, where we release the gas into a flat potential (i.e., a quench for which $\mathcal{Q}$ is an exact conserved quantity). We find values for the lower bound that are comparable to the quench into the cosine potential. For the density operator, the lower bound for the long time correlations is considerably larger than that for the MDF, being bounded by values of up to $\mathcal{O}(1)$. We thus see that our construction of $\mathcal{Q}$ acts to ensure that the system retains memory of its initial condition even at infinite time.

\section{ACKNOWLEDGMENTS}

We would like to warmly thank both Marcos Rigol and Neil Robinson for helpful discussions surrounding this work. The research herein was supported by the CMPMS Department, Brookhaven National Laboratory, in turn funded by the U.S. Department of Energy, Office of Basic Energy Sciences, under Contract No. DE-AC0298CH10886 (R. M. K.) and by the Netherlands Organization for Scientific Research (NWO) and the Foundation for Fundamental Research on Matter (FOM) (J. S. C. and G. P. B.).

\section{APPENDIX A: DESCRIPTION OF THE 1D BOSE GAS AND ITS POST-QUENCH DYNAMICS USING A NUMERICAL RENORMALIZATION GROUP}

\section{Application of the numerical renormalization group}

Our approach to describing the dynamics associated with the quantum quench of the gas is to employ a numerical renormalization group [58] that employs the eigenstates of the Lieb-Liniger model as a computational basis to

\section{Lower Bounds on $\chi_{k}$}

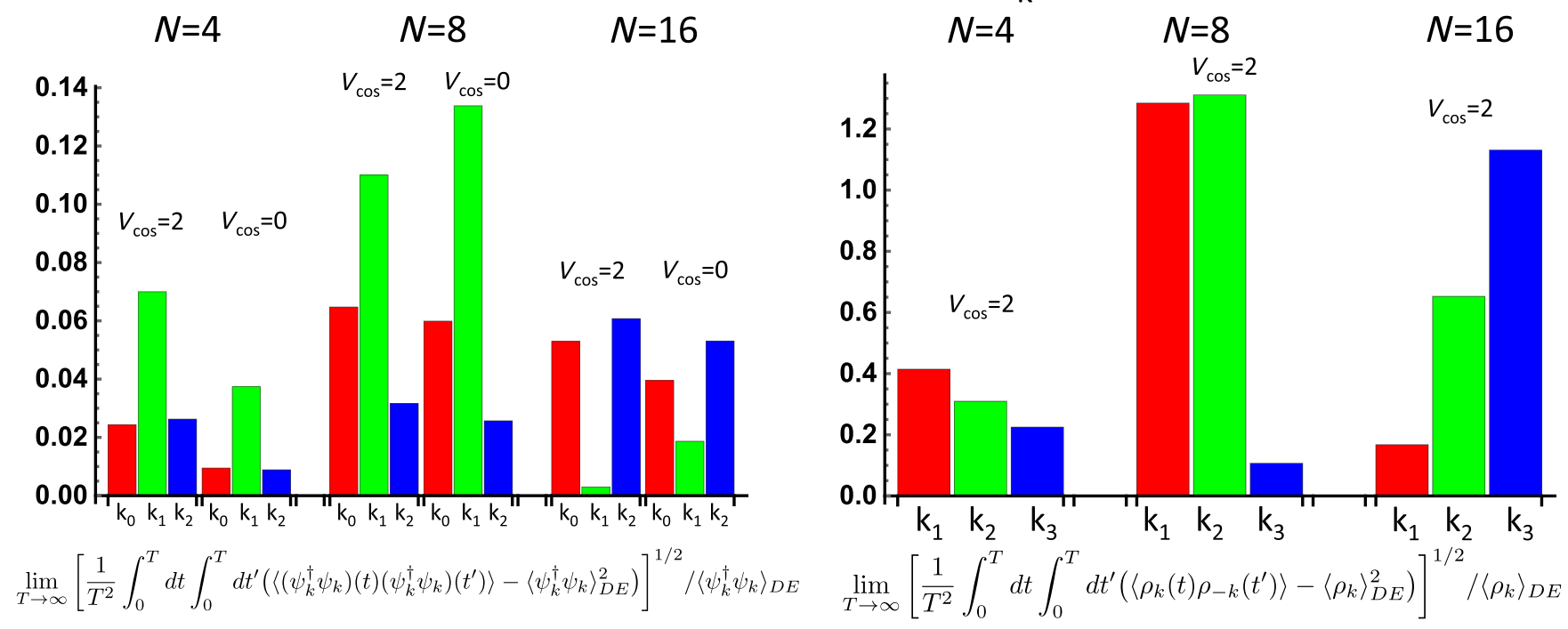

FIG. 9. The lower bounds on $\chi_{k}$ due to the effective charge $\mathcal{Q}$ for correlators involving the MDF and the density operators. Left-hand panel: We plot the lower bound on MDF correlations for two different quenches in a $c=10$ gas. In the first (the left sets of bars), we quench into a cosine potential $V_{\cos } \cos (2 \pi x / L)$ of amplitude $V_{\cos }=0.26 E_{F}$. In the second (the right set of bars), we quench into a flat potential, i.e., $V_{\text {cos }}=0$, where the post-quench Hamiltonian is then integrable. We present $\chi_{k}$ for three different system sizes $N=L=4,8$, and 16 and three different values of $k, k_{n}=2 \pi n / L, n=0,1$, and 2 . The initial state of the quench is given by the ground state of a gas in a parabolic potential of strength $\omega=2.4 / N$. Right-hand panel: We similarly plot the lower bound on density correlations. Here, we only consider the case of quenching into $V_{\cos }=2$, as $\chi_{k}$ for the density operator is identically zero in the absence of the breaking of translational invariance. We again compute the lower bound at three different system sizes and three different wave vectors $k_{1}, k_{2}$, and $k_{3}$. In both cases, we see no obvious dependence on system size. We believe that the fluctuations seen between different system sizes results from the particular construction of $\mathcal{Q}$ at any given system size. We construct $\mathcal{Q}$ to minimize time fluctuations of a particular initial condition rather than construct it to maximize its overlap with a particular observable as was done in Ref. [74]. 
determine the relatively low-lying eigenstates of the Bose gas in a one-body potential. This numerical renormalization group is built upon both ideas taken from Wilson's development of a numerical renormalization group used to study quantum impurity problems [75] and Zamolodchikov's numerical treatment of perturbed conformal field theories [62,63]. The use of the LiebLiniger basis as such a basis trades on our ability to be able to efficiently compute matrix elements of relevant operators such as the density operator exactly. While there are compact determinantal expressions for such matrix elements [76,77], their evaluation is still a nontrivial numerical task, and to this end, we use a set of computerized routines named ABACUS $[61,78,79]$. We have already demonstrated that we are able to perform the first step in our quench protocol: We have shown in Fig. 2 that we can accurately compute the ground state of the gas in the parabolic trap. In this figure, we plotted our numerical determination (black) of the density profile of a gas with $N=14$ particles in a system of length $L=14$ with an interaction parameter of $c=7200$ in a trap of strength $V_{\text {para }}=\omega_{0}^{2} x^{2} /(2 m)$ with $m \omega_{0}^{2} L^{2} / 2 E_{F}=10.36$ against the density profile determined analytically (red) by mapping these (nearly) hard-core bosons onto free fermions. The details of the analytic description of the gas in its hard-core limit are found in Appendix A12.

In the second step of the quench protocol, we released the gas into a one-body cosine potential,

$$
V_{\cos }(x)=A \cos \left(\frac{2 \pi n_{\cos } x}{L}\right)
$$

In order to compute the post-quench dynamics, we need to be able to describe not only the ground state in the cosine potential but also some large number of excited states. In our quench protocol, we take as our initial $t=0$ state the ground state of the gas in the parabolic potential, $\left|\psi_{\mathrm{GS}, \text { para }}\right\rangle$. If we can compute a wide range of eigenstates in the cosine potential, both ground and excited states, $\left|\psi_{\alpha, \cos }\right\rangle$, we can expand this initial state in terms of the post-quench basis:

$$
\left|\psi_{\mathrm{GS}, \mathrm{para}}\right\rangle=\sum_{\alpha} c_{\alpha}\left|\psi_{\alpha, \mathrm{cos}}\right\rangle
$$

Of course for this expansion to be exact, we would need to know all of the eigenstates of the gas in the cosine potential. We will instead settle for a determination of the postquench eigenbasis that allows us to include enough states so that $\sum_{\alpha}\left|c_{\alpha}\right|^{2}>0.99$. We note that after we determine the initial values of the overlap coefficients, $c_{\alpha}$, we proceed to normalize them so that their squares sum to 1 .

In computing the spectrum of states in the cosine potential, we employ the variant of the NRG discussed in Ref. [39]. The NRG in its plain vanilla formulation [58] can compute the spectrum of the low-lying states of the gas in the one-body potential [8]. But to accurately capture an appreciable fraction of the spectrum, we need to employ a sweeping routine [39] analogous to that used in the finite volume routine of the density matrix renormalization group [80,81].

In Fig. 10, we present results for the spectra of an $N=L=14$ gas in the hard-core limit $c=7200$. Here, we plot in black (right-hand side, r.h.s.) the numerical determination of the first 365 energy levels of the gas in a cosine potential. In red (left-hand side, l.h.s.), we plot the corresponding analytic determination of the levels. This analytic determination is possible by mapping the bosons to nearly free fermions who interact with a four-body term of strength $1 / c$. Again, the details of the analytics are found in Appendix A12. The difference between the numerics and the analytics here is less than $10^{-3}$ (in absolute units).

Once we have this expansion of our initial condition $\left|\psi_{\mathrm{GS}, \text { para }}\right\rangle$ in terms of the eigenstates in the cosine potential, we can readily determine the time evolution of the state post-quench:

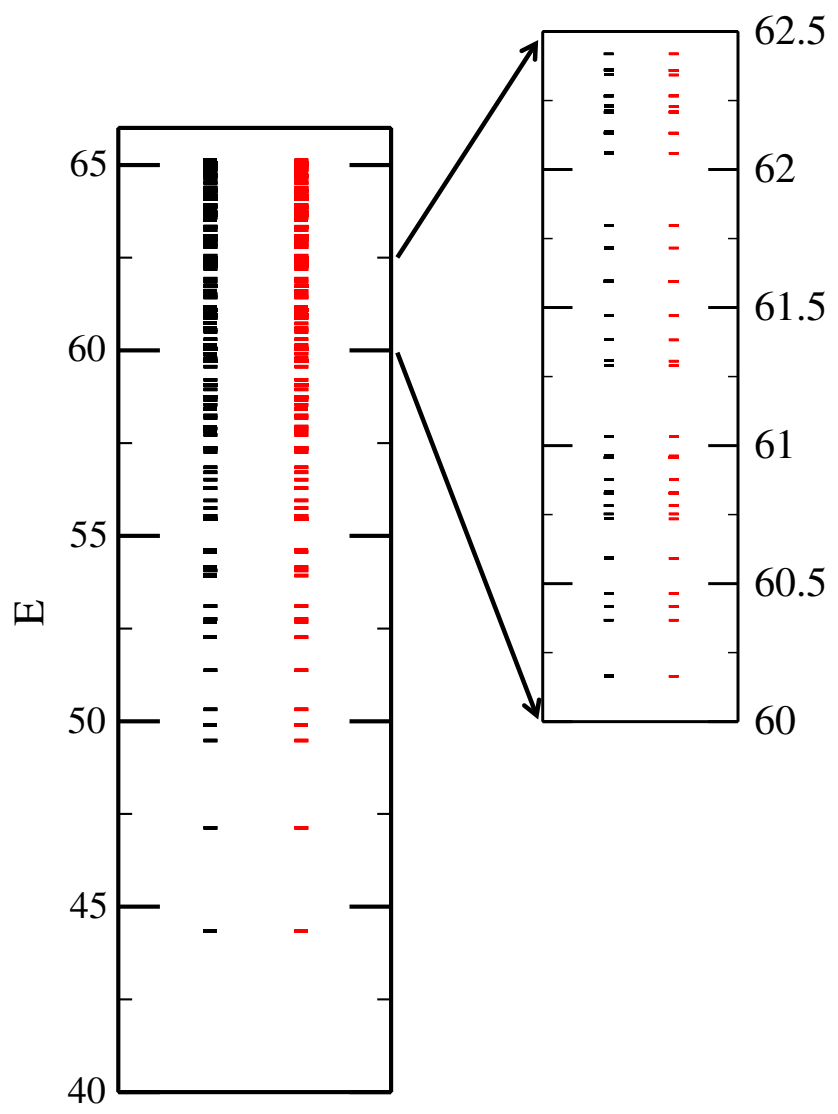

FIG. 10. A plot of the energy spectra for an $N=14$ gas with $c=7200$ in a cosine potential of amplitude $A / E_{F}=0.35$ (as in Fig. 2 of the main text). The analytic results are given in red, while in black are the corresponding numerics. On the r.h.s., we expand a range of energy with a dense number of states so as to better exhibit agreement between the numerics and the analytics. We can determine the first 365 states (up to energies of $E=65$ ) with accuracy of $10^{-3}$. 


$$
\left|\psi_{\mathrm{GS}, \mathrm{para}}\right\rangle(t)=\sum_{\alpha} c_{\alpha} e^{-i E_{\alpha} t}\left|\psi_{\alpha, \cos }\right\rangle
$$

We can track time evolution of the state to a point in time determined by the accuracy by which we can determine $E_{\alpha}$. If the accuracy to which we determine $E_{\alpha}$ is $\delta E_{\alpha}$, we can only track time evolution while $t \delta E_{\alpha} \ll 2 \pi$. After this inequality is violated, we are no longer able to trust the numerics. Concretely, we call a state $\left|\psi_{\alpha, \cos }\right\rangle$ dephased at time $t$ if $\delta E_{\alpha} t>0.01 \times 2 \pi$, and we conservatively will not track the time evolution beyond a point where the sum of states that are dephased have a weight exceeding 0.01, i.e., $\sum_{\alpha \in \text { dephased states }}\left|c_{\alpha}\right|^{2}>0.01$. Under this criterion, we can still track the dynamics out to considerable times. For the $N=14$ data in Fig. 2 of the main text, we can run out to times around $80 t_{F}$, while for the $N=8$ data in Figs. 3, 4, and 5 of the main text, we can run considerably longer, to $t \sim 6000 t_{F}$. While in Fig. 3 we present the time series for times close to this bound, we present in Fig. 11 the time series for the same sets of charges at shorter times, $t<100 t_{F}$.

With the time evolved state in hand, we are able to compute the time evolution of a number of observables and operators. Because we use the eigenstates of the LiebLiniger model minus a one-body potential, $\left|\psi_{\alpha, L L}\right\rangle$, as the computational basis of the NRG, the NRG gives any eigenstate in a one-body potential as a linear combination of such states:

$$
\left|\psi_{\text {one-body }}\right\rangle=\sum_{\alpha} b_{\alpha}\left|\psi_{\alpha, \mathrm{LL}}\right\rangle
$$

Thus, the dynamics of any operator whose matrix elements are known in the Lieb-Liniger basis can be determined. As one example, we plotted in Fig. 2 of the main text the time evolution post-quench of the density profile of the gas.

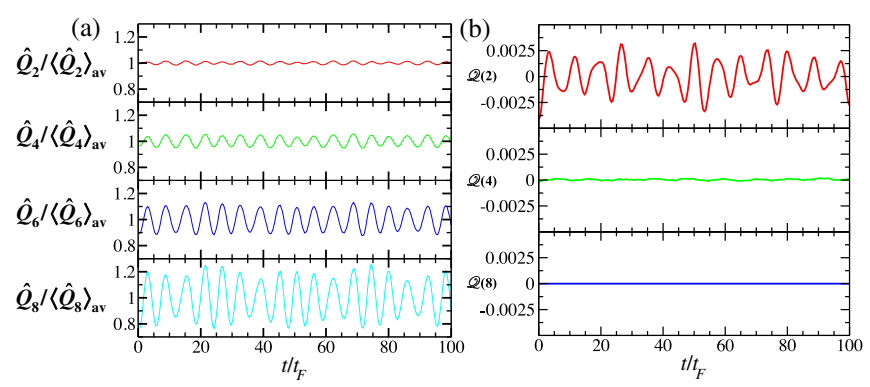

FIG. 11. (a) The post-quench time evolution of the same normalized Lieb-Liniger charges shown in Fig. 2 of the main text but at times $t<100 t_{F}$. (b) The post-quench time evolution of the sequence of effective charges, $\mathcal{Q}\left(N_{Q}\right)-a_{0} \mathrm{I}=\sum_{m=1}^{N_{Q}} a_{2 m} \hat{Q}_{2 m}$, shown in Fig. 2 for $N_{Q}=2,4$, and 8 for the same range of time.

\section{a. Error analysis of $\mathcal{Q}$ fluctuations}

One of the claims made in the text is that the effective charges $\mathcal{Q}$ that we construct have fluctuations that drop exponentially with the number $N_{Q}$ of Lieb-Liniger charges used in building them. For this to be a meaningful statement, we need to put a lower bound on the charge fluctuations arising from numerical error.

This error would arise from the dephasing errors that arise because we can only imperfectly determine the postquench energies. However, these errors are small. We run out to times where only postquench eigenstates representing $1 \%$ of the weight of the initial condition have dephased (defined as having a phase error greater than $1 \%$ of $2 \pi$ ), i.e., $1 \%$ of the weight of the state is dephased by $1 \%$.

This might then suggest that we find a lower bound of $10^{-4}$ on the fluctuations of the effective charges. However, the off-diagonal matrix elements of the effective charges are also very small. Thus, any error due to dephasing will be suppressed-fluctuations in the charges are due to offdiagonal matrix elements. So a lower bound on the error will be approximately the size of these off-diagonal matrix elements (also on the order of $10^{-4}$ ) times the square root of the number of off-diagonal matrix elements involved (square root because we assume the errors introduced by the off-diagonal matrix elements add in the fashion of a random walk) times the error due to dephasing, so approximately $10^{-6}$ to $10^{-7}$. This is roughly the lower bound we see on the charge fluctuations.

\section{Description of the gas in the cosine potential in the large $c$ limit}

In this appendix, we provide a description of the hardcore limit $(c \rightarrow \infty)$ of the Lieb-Liniger model defined on a ring of length $L$ in the presence of a cosine potential:

$$
\begin{aligned}
H_{B}= & -\sum_{i=1}^{N} \frac{1}{2 m} \frac{\partial^{2}}{\partial x_{i}^{2}}+c \sum_{i<j} \delta\left(x_{i}-x_{j}\right) \\
& +A \sum_{i=1}^{N} \cos \left(\frac{2 \pi n_{\cos }}{L} x_{i}\right) .
\end{aligned}
$$

The ability to do analytics in the hard-core limit will then serve as a check on our numerical results.

For $c \gg 1$, the system can be mapped onto a system of fermions with the Hamiltonian [82-84]

$$
\begin{aligned}
H_{F}= & -\sum_{i=1}^{N} \frac{1}{2 m} \frac{\partial^{2}}{\partial x_{i}^{2}}-\frac{2}{m^{2} c} \sum_{i<j} \delta^{\prime \prime}\left(x_{i}-x_{j}\right) \\
& +A \sum_{i=1}^{N} \cos \left(\frac{2 \pi n_{\cos }}{L} x_{i}\right),
\end{aligned}
$$


where in the dual picture we have an ultra-local interaction term of strength $1 / c$.

For $c \rightarrow \infty$, the fermions are noninteracting and the physics becomes effectively one-body [85]. We then must only solve the following single-body Schrödinger equation:

$$
-\frac{1}{2 m} \frac{\partial^{2}}{\partial x^{2}} \psi(x)+\cos \left(\frac{2 \pi n_{\cos }}{L} x\right) \psi(x)=E \psi(x) .
$$

This equation can be put in the standard form of the Mathieu equation,

$$
\frac{\partial^{2}}{\partial z^{2}} \psi(z)+(a-2 q \cos (2 z)) \psi(z)=0,
$$

if we identify

$$
\begin{gathered}
z=\frac{\pi n_{\mathrm{cos}}}{L} x, \\
q=\frac{A}{2}\left(\frac{L}{\pi n_{\mathrm{cos}}}\right)^{2}, \\
a=E\left(\frac{L}{\pi n_{\mathrm{cos}}}\right)^{2} .
\end{gathered}
$$

The Mathieu equation admits Floquet-type solutions of the form

$$
\begin{aligned}
& \psi_{\nu}^{1}(a, q, z)=e^{i \nu z} P(a, q, z), \\
& \psi_{\nu}^{2}(a, q, z)=\psi_{\nu}^{1}(a, q,-z)=e^{-i \nu z} P(a, q,-z),
\end{aligned}
$$

where $P(a, q, z)$ is a periodic function in $z$ of period $\pi$ (the same periodicity of the cosine term in the Mathieu equation). Here, $\nu=\nu(a, q)$, the Mathieu characteristic exponent function, is a function of $a$ and $q$. If $\nu$ is an integer, the second solution is not linearly independent and a new solution must be built (see Ref. [86]). In the following, we are interested only in noninteger solutions.

We are able to create linear combinations of the pairs of degenerate solutions for each triplet $\{a, q, \nu\}$. We focus on linear combinations that are even and odd in $z$ :

$$
\begin{aligned}
& \psi_{+\nu}(a, q, z)=\frac{\psi_{\nu}^{1}(a, q, z)+\psi_{\nu}^{2}(a, q, z)}{2}, \\
& \psi_{-\nu}(a, q, z)=\frac{\psi_{\nu}^{1}(a, q, z)-\psi_{\nu}^{2}(a, q, z)}{2 i} .
\end{aligned}
$$

The final step is to construct linear combinations of these solutions that satisfy the boundary conditions. This step amounts to the quantization of the values of $a$, i.e., the energy, and so $\nu$. For $N$ even, we need to impose antiperiodic boundary conditions on the single-particle solutions

$$
\psi(x+L)=-\psi(x) .
$$

This will still lead the eventual $N$-body wave function to be periodic, and it corresponds to the use of half-integer quantum numbers in constructing the solutions of the Bethe ansatz equations for $N$ even. To satisfy these boundary conditions, we choose $\nu$ to be

$$
\nu=\frac{2 n-1}{n_{\cos }}, \quad n=1,2 \ldots
$$

It is interesting to notice that for large enough $n$, the energy $a$ coming from the two Mathieu characteristic functions corresponding to $\psi_{-\nu}$ and $\psi_{+\nu}$ behaves as $a \sim n^{2}$, as would be expected when the kinetic energy of the state greatly exceeds its potential energy.

Multiparticle states are then constructed from these single-particle solutions according to Pauli's exclusion principle, remembering that there are two available states for each energy eigenvalue $(\nu$ and $-\nu)$. In comparing to the analytic solutions of the gas in the cosine potential, we perform our numerics not at $c=\infty$ but at a large finite value of $\mathrm{c}(c=7200)$. We thus consider perturbative corrections in $1 / c$ to the hard-core limit. As the $1 / c$ correction to the Hamiltonian,

$\delta H_{F}=-\frac{2}{m^{2} c} \int d x d x^{\prime} V\left(x-x^{\prime}\right) \psi^{\dagger}(x) \psi^{\dagger}\left(x^{\prime}\right) \psi\left(x^{\prime}\right) \psi(x)$,

treats two particles at a time, we can first compute the correction in energy for the two-particle case, and then for the $N$-particle case simply by adding the $\left(\begin{array}{c}N \\ 2\end{array}\right)$ contributions coming from all possible particle pairs.

\section{APPENDIX B: ANALYTIC CONSTRUCTION OF CHARGES IN THE HARD-CORE LIMIT}

\section{General discussion of analytic construction}

We have shown that we can construct numerically quasiconserved quantities formed as linear combinations of Lieb-Liniger charges, where the quality of the conservation is controlled by the number of charges in the combination. But while we have a concrete numerical construction of these new quasicharges, we have only a minimal analytic understanding of why such charges exist. Is this happenstance or can we provide something more solid? The answer is that we can, and it is the aim of this appendix.

The basic idea behind this is to show that we can systematically construct charges of the form $\mathcal{Q}=\sum_{i} a_{i} \hat{Q}_{i}$ that zero out low-lying matrix elements that would otherwise lead them to have a nontrivial time dependence. That $\mathcal{Q}$ has a time dependence at all is because of the one-body potential $V(x)$ in the post-quench Hamiltonian: 


$$
\begin{aligned}
H_{\text {post-quench }} & =H_{\mathrm{LL}}+V_{\text {cosine }} \\
V_{\text {cosine }} & =\int_{0}^{L} d x V(x) \hat{\rho}(x),
\end{aligned}
$$

where for us $V(x)=A \cos \left(2 \pi n_{\cos } x / L\right)$. We can rewrite this term in terms of the Fourier components of $V(x)$ and the density operator $\hat{\rho}(x)$ :

$$
\begin{aligned}
\int_{0}^{L} d x V(x) \hat{\rho}(x) & =\sum_{k} V_{k} \hat{\rho}_{k} \\
& =\frac{1}{2}\left(\hat{\rho}_{k_{n_{\mathrm{cos}}}}+\hat{\rho}_{-k_{n_{\mathrm{cos}}}}\right),
\end{aligned}
$$

where $\hat{\rho}_{k_{n}}=\sum_{q} \psi_{q+k_{n}}^{\dagger} \psi_{q}$ with $k_{n}=2 \pi n / L$.

The time dependence of $\mathcal{Q}(t)$ can be written as a power series in time $t$ via the Baker-Campbell-Hausdorff formula:

$$
\begin{aligned}
\mathcal{Q}(t) & =e^{i V_{\text {cosine }} t} \mathcal{Q} e^{-i V_{\text {cosine }} t} \\
& =\mathcal{Q}+i t C_{1}+\frac{(i t)^{2}}{2 !} C_{2}+\frac{(i t)^{3}}{3 !} C_{3}+\cdots \\
C_{1} & =\left[\sum_{k} V_{k} \hat{\rho}_{k}, \mathcal{Q}\right] ; \quad C_{n \geq 2}=\left[\sum_{k} V_{k} \hat{\rho}_{k}, C_{n-1}\right] .
\end{aligned}
$$

What we now argue is that we can systematically zero out all low-energy matrix elements (below some designated cutoff) of the first term involving the commutator of the one-body potential with $\mathcal{Q}(t)$. This results in a charge $\mathcal{Q}$ that has a $t^{2}$ (and higher) time dependence on the lowenergy Hilbert space. However, this higher-order dependence is only nominal. What we observe is that for a cosine one-body potential, zeroing out first-order matrix elements also zeros out a large number of matrix elements from higher-order commutators that arise from one-particle-hole processes. To keep things tractable in this construction, we mostly focus on the $c=\infty$ limit where there is no more than one particle-hole process.

To understand why higher orders remain zeroed out, we first need to describe the Hilbert space as spanned by the Lieb-Liniger eigenstates in a bit more detail. An eigenstate of the Lieb-Liniger model is described by $N$-rapidities, $\lambda_{i}$,

$$
|s\rangle=\left|\lambda_{1}, \ldots, \lambda_{N}\right\rangle=\left|I_{1}, \ldots, I_{N}\right\rangle,
$$

which in turn are determined by $N$-integers (or halfintegers) via the Bethe ansatz equations:

$$
\begin{aligned}
2 \pi I_{i} & =L \lambda_{i}+\sum_{j \neq i} \phi\left(\lambda_{i}-\lambda_{j}\right), \\
\phi(\lambda) & =2 \tan ^{-1}\left(\frac{\lambda}{c}\right) .
\end{aligned}
$$

We use the notion of these quantum numbers both to delineate the zeroed-out portion of the Hilbert space and to describe how it changes under higher-order processes.

Let us now construct the effective charge

$$
\mathcal{Q}=\sum_{i=1}^{N_{Q}} a_{i} \hat{Q}_{i},
$$

by defining it to have the following property: If the integers characterizing $|s\rangle$ and $\left|s^{\prime}\right\rangle$ are all such that

$$
\left|I_{i}\right|, \quad\left|I_{i}{ }^{\prime}\right| \leq N_{\max },
$$

then the following matrix element vanishes:

$$
\left.\left\langle s\left|\left[\sum_{k} V_{k} \hat{\rho}_{k}, \mathcal{Q}\right]\right| s^{\prime}\right\rangle=\langle s| C_{1}, \mathcal{Q}\right]\left|s^{\prime}\right\rangle=0 .
$$

This condition amounts to insisting that

$$
\left(\mathcal{Q}\left(s^{\prime}\right)-\mathcal{Q}(s)\right)\left\langle s\left|V_{\text {cosine }}\right| s^{\prime}\right\rangle=0,
$$

where $\mathcal{Q}(s)$ is the action of $\mathcal{Q}$ on the state $|s\rangle$, i.e., $\mathcal{Q}|s\rangle=\mathcal{Q}(s)|s\rangle$.

Provided we are willing to include enough Lieb-Liniger charges in $\mathcal{Q}$ (i.e., choose $N_{Q}$ large enough), we can always find a $\mathcal{Q}$ satisfying Eq. (B8), as the collection of constraints in Eq. (B8) form a set of homogeneous linear equations:

$$
\begin{aligned}
& \sum_{i=1}^{N_{Q}} a_{i}\left(Q_{i}\left(s^{\prime}\right)-Q_{i}(s)\right)=0, \\
& \quad \text { for all }|s\rangle,\left|s^{\prime}\right\rangle \text { satisfying Eq. (B6). }
\end{aligned}
$$

The number of charges $N_{Q}$ we need to include to be able to find a nontrivial solution behaves as $N_{Q}=N_{\max }+2$, a number that is effectively proportional to the $\log$ of the size of Hilbert space.

We now suppose we have constructed a $\mathcal{Q}$ where a block of states of its commutator with $V_{\text {cosine }}$ has been zeroed out-see the top square in Fig. 12 for a graphical representation of this. But now how does this zero block fare when we consider higher-order commutators,

$$
\left\langle s\left|\left[V_{\text {cosine }}, C_{l}\right]\right| s^{\prime}\right\rangle,
$$

that appear in the Taylor series of $\mathcal{Q}(t)$ ? Roughly speaking, this block does not immediately disappear at higher order but rather only shrinks linearly with the order of the commutator. The $l+1$ th-order commutator, $\left.\langle s| V_{\text {cosine }}, C_{l}\right]\left|s^{\prime}\right\rangle$, will have nonzero matrix elements between two states, $|s\rangle$ and $\left|s^{\prime}\right\rangle$, provided that their quantum numbers satisfy

$$
\left|I_{i}\right|, \quad\left|I_{i}{ }^{\prime}\right| \leq N_{\max }-(l-1) n_{\cos } .
$$


Thus, for every order in the perturbative expansion, we shrink the block of zero matrix elements by $n_{\text {cos }}$.

We can see this simply for matrix elements of the second-order commutator. Suppose then that $|s\rangle$ and $\left|s^{\prime}\right\rangle$ are states whose quantum numbers, $\left\{I_{i}\right\}$ and $\left\{I_{i}^{\prime}\right\}$, satisfy

$$
\left|I_{i}\right|, \quad\left|I_{i}{ }^{\prime}\right| \leq N_{\max }-n_{\cos }
$$

Then, the matrix element $\left\langle s\left|C_{2}\right| s^{\prime}\right\rangle$ equals

$$
\left\langle s\left|C_{2}\right| s^{\prime}\right\rangle=\left\langle s\left|V_{\text {cosine }} C_{1}-C_{1} V_{\text {cosine }}\right| s\right\rangle .
$$

Now, the action of $V_{\text {cosine }}$ on $|s\rangle$ is to give a state $V_{\text {cosine }}|s\rangle=\left|\tilde{I}_{1}, \ldots, \tilde{I}_{N}\right\rangle$ whose quantum numbers must satisfy (using that the action of $V_{\text {cosine }}$ is to change one quantum number by $\left.\pm n_{\cos }\right)$

$$
\left|I_{i}\right|, \quad\left|I_{i}^{\prime}\right| \leq N_{\max } .
$$

But, by construction, the matrix elements of $C_{1}$ between such a state and $|s\rangle$ are zero. Hence, $C_{2}$ has a reduced block of zeros. This continues on to higher order in an iterative fashion. At $c=\infty$ the situation is actually even better than this argument would indicate. In fact the block of zero matrix elements for $C_{n>1}$ only shrinks to states with quantum numbers less than

$$
N_{\max }-\left(n_{\cos }\left\lfloor\frac{n}{2}\right\rfloor-1\right) .
$$

This result for the $c=\infty$ case of how the zeroed out matrix elements remain for the higher order commutators is pictured in Fig. 12.

Having outlined how we can construct quasiconserved charges analytically, we now numerically test this quasiconservation. To perform this test, we construct a sequence of effective charges $\left\{\mathcal{Q}_{N_{\max }}\right\}$, defined by a sequence of maximal quantum numbers, $N_{\max }$. A charge $\mathcal{Q}_{N_{\max }}$ is defined by its first-order commutator $C_{1}=\left[V_{\text {cosine }}, \mathcal{Q}_{N_{\max }}\right]$ having no nonzero matrix elements involving any two states $|s\rangle,\left|s^{\prime}\right\rangle$ whose quantum numbers are less than or equal to $N_{\max }$. Such a charge will have to satisfy a number of constraints of the type found in Eq. (B9). As we have already stated, there are $N_{\max }+1$ such constraints. As such we form $\mathcal{Q}_{N_{\max }}$ as a linear combination of $N_{Q}\left(N_{\max }\right)=$ $N_{\max }+2$ charges:

$$
\begin{aligned}
\mathcal{Q}_{N_{\max }} & =a_{0}+\sum_{i=1}^{N_{Q}\left(N_{\max }\right)} a_{i} \frac{\hat{Q}_{2 i}}{\left\langle\hat{Q}_{2 i}\right\rangle_{\mathrm{av}}} ; \\
1 & =\sum_{i=1}^{N_{Q}\left(N_{\max }\right)}\left|a_{i}\right|^{2} ; \\
\left\langle\hat{Q}_{i}\right\rangle_{\mathrm{av}} & =\frac{1}{T} \int_{0}^{T} d t\left\langle\hat{Q}_{i}(t)\right\rangle ;
\end{aligned}
$$
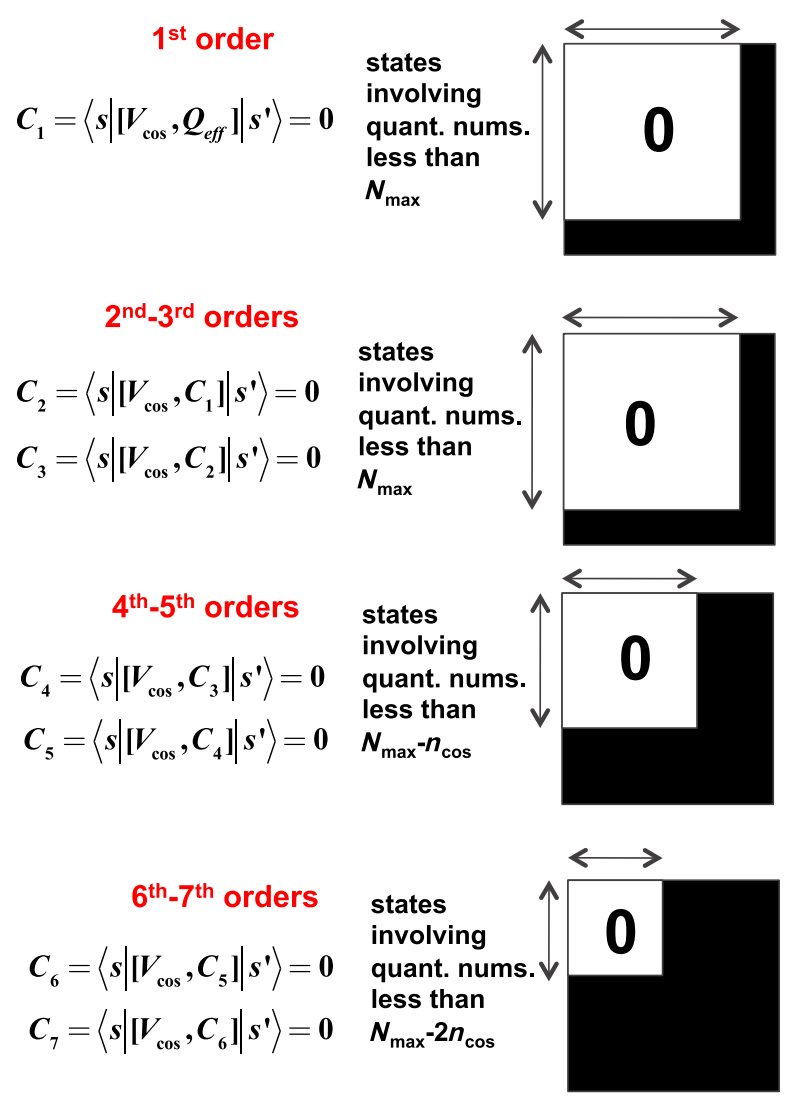

FIG. 12. Here we show at $c=\infty$ how the matrix elements zeroed out for the commutator $C_{1}$ remain zero for the higher order commutators $C_{n>1}$. In the top block we graphically display how the matrix elements of $C_{1}$ are zeroed out for states with quantum numbers less than $N_{\max }$. In the second block, we show that the matrix elements of the second and third order commutators $C_{2}$ and $C_{3}$ are also zero for this same set of states. It is only for the fourth and fifth order commutators, $C_{4}$ and $C_{5}$, that the set of states with zero matrix elements begins to shrink to states with quantum numbers less than $N_{\max }-n_{\cos }$ (as pictured in the third block). The set of states with zero matrix elements on $C_{6}$ and $C_{7}$ shrinks further to states with quantum numbers less than $N_{\max }-2 n_{\cos }$. This shrinkage in the set of states by steps of $n_{\text {cos }}$ continues at higher order, one step for every two orders of commutators.

i.e., we include the minimal number of Lieb-Liniger charges $\hat{Q}_{i}$ so that the null space of the set of linear equations in Eq. (B9) has dimension 1. As in the main body of the text, we normalize the charges $\hat{Q}_{i}$, with respect to their time average following a particular quench.

In Fig. 13, we provide two tests of the quality of the conservation of the charge $\mathcal{Q}$ as a function of $N_{\max }$. In panel (c) of Fig. 13, we consider the dynamics of $\mathcal{Q}_{N_{\max }}(t)$ postquench in our standard quantum quench protocol (preparing the gas in a parabolic potential and releasing it into a cosine potential). We see that the fluctuations in time of $\mathcal{Q}_{N_{\max }}(t)$ post-quench decrease exponentially with $N_{\max }$.

However, the real test of the quality of quasiconservation of the sequence of $\left\{\mathcal{Q}_{N_{\max }}\right\}$ is to be found in the size of 

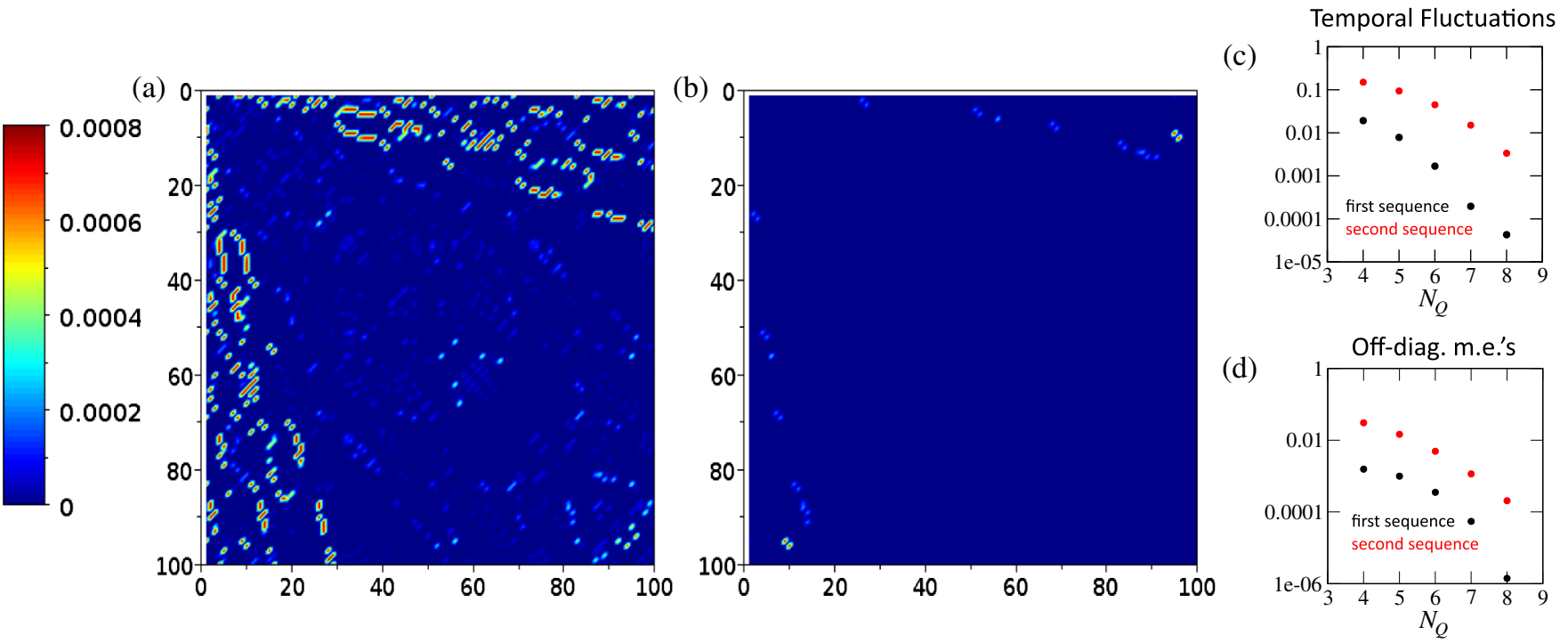

FIG. 13. The magnitude of the off-diagonal matrix elements of the analytically constructed effective charges composed from (a) four Lieb-Liniger charges, $\mathcal{Q}_{N_{\max }}(4)$, and (b) eight Lieb-Liniger charges, $\mathcal{Q}_{N_{\max }}$ (8). (c) The size of the post-quench temporal fluctuations of the effective charges $\mathcal{Q}_{N_{\max }}\left(N_{Q}\right)$ as a function of the number of charges in the linear combination. Here, the quench is performed by preparing an $N=8, c=7200$ gas in a parabolic potential of strength $m \omega_{0}^{2} L^{2} / 2 E_{F}=6.48$ released into a cosine potential of strength $A=1$. We show the size of the temporal fluctuations for two sequences of effective charges, the first (in black) constructed from LiebLiniger charges, $Q_{2 m}, m=1, \ldots, 8$ and the second (in red) constructed from $Q_{2 m}, m=9, \ldots, 16$. (d) The size of the off-diagonal matrix elements of these same two sequences of $\mathcal{Q}_{N_{\max }}$ as a function of the number $N_{Q}$ of Lieb-Liniger charges in the linear combination.

their off-diagonal matrix elements on the low-energy postquench Hilbert space. To this end, we display the size of these off-diagonal matrix elements in panels (a) and (b) of Fig. 13. In this figure, we show two intensity plots corresponding to $N_{\max }=4$ and 8 . We see that the charge $\mathcal{Q}$ built for $N_{\max }=8$ has considerably smaller off-diagonal terms than does $\mathcal{Q}$ for $N_{\max }=4$. This is quantified in panel (d) of Fig. 13 where we plot the average magnitude of the offdiagonal matrix elements of $\mathcal{Q}_{N_{\max }}$ as a function of $N_{\max }$. We see that it drops exponentially with the number of charges.

\section{a. Equivalence of the two constructions of the charges}

We have now demonstrated an analytic method to construct effective charges $\mathcal{Q}$. But what is the relationship between these and those derived numerically from a particular quench protocol? We show that, in fact, they do coincide. In order to demonstrate this, we first fix $N_{\max }$. While we have argued that we only need $N_{Q}=N_{\max }+2$ charges to find a single nontrivial solution of the linear equations in Eq. (B9), we consider these equations with $N_{Q}=2 N_{\max }$ charges - and so the linear equations now have a null space of dimension $N_{\max }-2$. We then proceed to find this null space. Having done this, we compute numerically (as in the main text) the effective charge built from $N_{Q}=2 N_{\text {max }}$ Lieb-Liniger charges that arises from minimizing the post-quench temporal fluctuations. We then ask whether this charge (or, more precisely, the vector of its coefficients, $\left\{a_{i}\right\}_{i=1}^{N}$ ) lies in the null space coming from analytically building the $\mathcal{Q}$ 's. We find that it does lie in the null space as $N_{\max }$ grows. This result is summarized in Table I. In particular, the column labeled "Projection" gives the projection of the normalized vector of coefficients $\left\{a_{i}\right\}_{i=1}^{N}$ into the null space (a value of 1 indicates the numerical charge lies entirely in the null space). We see that as $N_{\max }$ increases, this projection increases quickly to its maximum possible value. Thus, we conclude that the two methods yield the same effective charge $\mathcal{Q}$.

\section{Estimating the temporal variation of $\mathcal{Q}(t)$}

In this section, we estimate the quality of the conservation of the charges $\mathcal{Q}(t)$ that we have constructed in the previous section. We do so for both weak and strong amplitudes of the post-quench cosine potential.

To determine the magnitude of the time variation in $\mathcal{Q}(t)$ following the quench, we first express the initial condition, $\left|\psi_{\text {para }}\right\rangle$, in terms of the post-quench eigenbasis $\left|\psi_{\alpha, \cos }\right\rangle$ :

$$
\left|\psi_{\text {para }}\right\rangle=\sum c_{\alpha}\left|\psi_{\alpha, \cos }\right\rangle,
$$

and then, in turn, express $\mathcal{Q}(t)$ in terms of matrix elements of $\mathcal{Q}$ in this basis:

TABLE I. Degree to which numerical $\mathcal{Q}$ lies in null space of analytic $\mathcal{Q}$ 's for a $c=7200, N=L=8$ gas.

\begin{tabular}{lccc}
\hline \hline$N_{\max }$ & $N_{Q}$ & Dimension of null space & Projection \\
\hline 2 & 4 & 1 & 0.282 \\
3 & 6 & 2 & 0.718 \\
4 & 8 & 3 & 0.982 \\
\hline \hline
\end{tabular}




$$
\begin{aligned}
\mathcal{Q}(t) & =\sum_{\alpha \beta} c_{\alpha}^{*} c_{\beta}\left\langle\psi_{\alpha, \cos }|\mathcal{Q}(t)| \psi_{\beta, \cos }\right\rangle \\
& =\sum_{\alpha \beta} c_{\alpha}^{*} c_{\beta} e^{-i\left(E_{\beta}-E_{\alpha}\right) t}\left\langle\psi_{\alpha, \cos }|\mathcal{Q}| \psi_{\beta, \cos }\right\rangle
\end{aligned}
$$

We have argued in Appendix B 1 that the construction of $\mathcal{Q}(t)$ is such that the time dependence (at least up to some order in time) of the low-energy off-diagonal matrix elements of $\mathcal{Q}(t)$ is zeroed out. This result implies that some of the terms in the above expansion will be either zero or at least small. But which ones, and what weight do they carry? The matrix elements we have zeroed out are not in the post-quench basis but in the Lieb-Liniger eigenbasis, the eigenbasis of the gas without a one-body potential. To see the effects of this zeroing out, we expand $\left|\psi_{\alpha, \cos }\right\rangle$ in terms of this basis:

$$
\left|\psi_{\alpha, \cos }\right\rangle=\sum_{I_{1}>\cdots>I_{N}} c_{\alpha, I_{i}}\left|I_{1}, \ldots, I_{N}\right\rangle
$$

where the state $\left|I_{1}, \ldots, I_{N}\right\rangle$ is constructed according to Eqs. (B4) and (B5). We then, in turn, rewrite $\mathcal{Q}(t)$ in terms of matrix elements involving this Lieb-Liniger basis:

$$
\mathcal{Q}(t)=\sum_{\substack{\alpha, \beta \\ I_{1}, \cdots \rightarrow I_{N} \\ J_{1}>\cdots>J_{N}}} c_{\alpha}^{*} c_{\beta} c_{\alpha, I_{i}}^{*} c_{\beta, J_{i}}\left\langle I_{i}|\mathcal{Q}(t)| J_{i}\right\rangle .
$$

From our construction of $\mathcal{Q}$, we see that the matrix elements involving states $\left|I_{1}, \ldots, I_{N}\right\rangle$ and $\left|J_{1}, \ldots, J_{N}\right\rangle$ with $\left|I_{i}\right|,\left|J_{i}\right| \leq N_{\max }$ will vanish. Because all states are normalized, we know that

$$
1=\sum_{\substack{\alpha, \beta \\ I_{1}>\cdots, I_{N} \\ J_{1}>\cdots>J_{N}}}\left|c_{\alpha}^{*} c_{\beta} c_{\alpha, I_{i}}^{*} c_{\beta, J_{i}}\right|^{2}
$$

To estimate how much of the time dependence of $\mathcal{Q}(t)$ has been eliminated, we want to compute the truncated sum,

$$
W_{\text {elim }}=\left.\sum_{\substack{\alpha, \beta \\ I_{1}>\cdots, J_{N} \\ J_{1}>\cdots, J_{N}}}\left|c_{\alpha}^{*} c_{\beta} c_{\alpha, I_{i}}^{*} c_{\beta, J_{i}}\right|^{2}\right|_{\left|I_{i}\right|,\left|J_{i}\right| \leq N_{\max }} \cdot
$$

The fluctuations in $\mathcal{Q}(t)$ will then go as $1-W_{\text {elim }}$.

In general, estimating $W_{\text {elim }}$ is difficult. However, we are able to do so in the limits of a weak and strong cosine potential. Because we are working at $c=\infty$, the pre- and post-quench wave functions of the $\mathrm{N}$-particle gas can be described as Slater determinants of single-particle states. Pre-quench, these single-particle states, $\left|\chi_{n}\right\rangle$, are associated with wave functions, $\chi_{n}(x)$, given in terms of Hermite polynomials: $\chi_{n}(x)=\frac{1}{\sqrt{2^{n} n !}}\left(\frac{m \omega_{0}}{\pi}\right)^{1 / 4} e^{-m \omega_{0} x^{2} / 2} H_{n}\left(x \sqrt{m \omega_{0}}\right)$.

Post-quench, the single-particle states $\left|\psi_{\nu}\right\rangle$ have wave functions given by Mathieu functions [with $\nu=$ $\pm(2 n+1) / n_{\text {cos }}$ ], as discussed in Appendix A 2 [see Eqs. (A14) and (A17)]. The $N$-particle eigenstates can then be denoted by

$$
\left|\psi_{\text {para }}\right\rangle=\left|\chi_{I_{1}}, \ldots, \chi_{I_{N}}\right\rangle
$$

pre-quench and

$$
\left|\psi_{\alpha, \cos }\right\rangle=\left|\psi_{\nu_{1}}, \ldots, \psi_{\nu_{N}}\right\rangle
$$

post-quench. The overlap between pre- and post-quench eigenstates can then be written as a sum over products of single-particle overlaps

$$
c_{\alpha}=\left\langle\psi_{\alpha, \cos } \mid \psi_{\text {para }}\right\rangle=\sum_{P \in S_{N}} \operatorname{sign}(P) \prod_{j=1}^{N}\left\langle\chi_{j} \mid \psi_{\nu_{P_{j}}}\right\rangle .
$$

Now, we specialize to the weak and strong cosine potential cases.

\section{a. Weak cosine amplitudes}

For weak amplitudes of the cosine potential, the postquench single-particle wave functions are approximately plane waves:

$$
\psi_{\nu}(x) \approx \frac{1}{\sqrt{L}} e^{i\left(\pi n_{\cos } \nu x\right) / L},
$$

and the $N$-particle states $\left|\psi_{\alpha, \text { cos }}\right\rangle$ are approximately LiebLiniger eigenstates:

$$
\left|\psi_{\alpha, \cos }\right\rangle \approx\left|I_{1}, \ldots, I_{N}\right\rangle .
$$

The sum $W_{\text {elim }}$ simplifies in this case to

$$
\begin{aligned}
W_{\text {elim }} & =\left.\sum_{\substack{I_{1}>\cdots>I_{N} \\
J_{1}>\cdots>J_{N}}}\left|c_{I_{1}, \ldots, I_{N}} c_{J_{1}, \ldots, J_{N}}\right|^{2}\right|_{\left|I_{i}\right|,\left|J_{i}\right| \leq N_{\max }} \\
& =\left(\sum_{N_{\max } \geq I_{1}>\ldots>I_{N} \geq-N_{\max }}\left|c_{I_{1}, \ldots, I_{N}}\right|^{2}\right)^{2} \\
& \equiv X_{\text {elim }}^{2},
\end{aligned}
$$

where we have introduced the variable $X_{\text {elim }}$. Because the single-particle overlaps describing the $N$-particle coefficients, $c_{\alpha}$, in Eq. (B20) are given by

$$
\left\langle\chi_{n} \mid \psi_{\nu_{j}}\right\rangle=i^{n} \sqrt{\frac{2 \pi}{m \omega_{0} L}} \chi_{n}\left(\frac{\pi \nu_{j} n_{\cos }}{L \sqrt{m \omega_{0}}}\right),
$$


we can reduce the sum $X_{\text {elim }}$ to

$$
\begin{aligned}
X_{\text {elim }}= & \left(\frac{2 \pi}{\sqrt{\pi m \omega_{0}} L}\right)^{N} \sum_{N_{\max } \geq I_{1}>\cdots>I_{N} \geq-N_{\max }} e^{-\sum_{i=1}^{N}\left(k_{i}^{2} / m \omega_{0}\right)} \\
& \times \sum_{P, P^{\prime}} \operatorname{sign}\left(P P^{\prime}\right) \prod_{n=0}^{N-1} H_{n}\left(\frac{k_{P_{i}}}{\sqrt{m \omega_{0}}}\right) H_{n}\left(\frac{k_{P_{i}^{\prime}}}{\sqrt{m \omega_{0}}}\right),
\end{aligned}
$$

where $P$ and $P^{\prime}$ are permutations of the integers $\left(I_{1}, \ldots, I_{N}\right)$. In the above equation, the off-diagonal terms of the sum, $\sum_{P, P^{\prime}}$ (i.e., those terms involving different permutations, $P_{i} \neq P_{i}{ }^{\prime}$ ), are at most of order $e^{-2 k_{\max }^{2} /\left(m \omega_{0}\right)}\left(k_{\max }^{2} /\left(m \omega_{0}\right)\right)^{4 N-4}$, and so can be ignored in comparison to the diagonal terms, which take the form

$$
1-\text { const } \times e^{-k_{\max }^{2} /\left(m \omega_{0}\right)}\left(k_{\max } /\left(m \omega_{0}\right)\right)^{2 N-3} .
$$

Thus, the leading-order correction to the diagonal terms (which is what we care about in determining how much weight is left over as encoded by $1-W_{\text {elim }}$ ) is much larger than the off-diagonal terms, which we henceforth ignore. We can then rewrite $X_{\text {elim }}$ by converting the sums to integrals:

$X_{\text {elim }}=\prod_{n=1}^{N-1} \frac{1}{\sqrt{m \omega_{0} \pi} 2^{n} n !} \int_{-k_{\max }}^{k_{\max }} d k_{i} H_{n}^{2}\left(\frac{k_{i}}{\sqrt{m \omega_{0}}}\right) e^{-\left(k_{i}^{2} / m \omega_{0}\right)}$,

where $k_{\max }=k_{\max }\left(N_{Q}\right)=2 \pi N_{\max }\left(N_{Q}\right) / L=2 \pi\left(N_{Q}-2\right) / L$. This can then readily be computed to be

$$
X_{\mathrm{elim}}=1-\frac{e^{-\Lambda\left(N_{Q}\right)^{2}}}{\sqrt{\pi}} \sum_{n=0}^{N-1} \frac{2^{n} \Lambda\left(N_{Q}\right)^{2 n-1}}{n !},
$$

where $\Lambda\left(N_{Q}\right)=k_{\max }\left(N_{Q}\right) / \sqrt{m \omega_{0}}$.

We then see that $1-X_{\text {elim }}^{2}$ goes as an exponential in $N_{\max }^{2}$ (and so $N_{Q}$ ), thus implying the fluctuations in $\mathcal{Q}(t)$ are suppressed exponentially in $N_{Q}^{2}$.

\section{b. Strong cosine amplitudes}

We now turn to the case of strong cosine amplitudes. We see that the fluctuations are expected to die much more slowly with $N_{Q}$ than in the weak case.

In this limit, we necessarily treat the $N$-particle post-quench wave functions as antisymmetrized products of Mathieu functions labeled by $\left\{\nu_{i}\right\}$, i.e., $\left|\psi_{\alpha, \cos }\right\rangle=$ $\left|\nu_{1}, \ldots, \nu_{N}\right\rangle$. The overlap $c_{\alpha, I_{i}}$ is then given by

$$
c_{\alpha, I_{i}}=\sum_{P} \operatorname{sign}(P) \prod_{i=1}^{N}\left\langle\nu_{i} \mid n_{P_{i}}\right\rangle,
$$

where $\left\langle\nu_{i} \mid n_{P_{i}}\right\rangle$ is the overlap between a single-particle Mathieu function associated with $\nu_{i}$ and the plane wave $n_{P_{i}}$. There is no closed-form expression for this overlap (as far as we know). However, for the purposes of this section, we use the following approximate:

$$
\langle\nu \mid n\rangle \approx \Theta\left(N_{\nu}-|n|\right) c_{\nu n}, \quad \text { if } \frac{\nu n_{\cos }}{2} \leq N_{A},
$$

where $N_{A}=(L \sqrt{2 m A} / 2 \pi)$ and where the coefficients $c_{\nu n}$ satisfy $\sum_{n=-N_{\nu}}^{n=N_{\nu}}\left|c_{\nu n}\right|^{2}=1$. This estimate says that the expansion of a Mathieu function in terms of plane waves only has a finite number of terms, $2 N_{\nu}$, provided $\nu$ is below a bound set by $N_{A}$. Beyond this bound, Mathieu functions become plane-wave-like (their kinetic energy is much greater than their potential energy), and their Fourier expansion changes to one consisting of a single plane wave. The coefficients $c_{\nu n}$ in this expansion oscillate between positive and negative amplitudes with (roughly) uniform amplitude. While there are Fourier coefficients of the Mathieu functions with modes beyond $N_{\nu}$, these coefficients are exponentially small in comparison to those for $|n| \leq N_{\nu}$.

We now evaluate $\sum_{I_{i}}\left|c_{\alpha, I_{i}}\right|^{2}$ :

$$
\begin{aligned}
\sum_{I_{i}}\left|c_{\alpha, I_{i}}\right|^{2} & =\sum_{I_{i}, P, P^{\prime}} \operatorname{sign}(P) \operatorname{sign}\left(P^{\prime}\right) \prod_{i=1}^{N}\left\langle\nu_{i} \mid n_{P_{i}}\right\rangle\left\langle n_{P_{i}^{\prime}} \mid \nu_{i}\right\rangle \\
& \approx \sum_{-N_{\max } \leq I_{1}<\cdots<I_{N} \leq N_{\max }} \sum_{P} \prod_{i=1}^{N}\left|\left\langle\nu_{i} \mid n_{P_{i}}\right\rangle\right|^{2} \\
& \approx \prod_{i=1}^{N} \sum_{I_{i}=-N_{\max }}^{N_{\max }}\left|\left\langle\nu_{i} \mid n_{P_{i}}\right\rangle\right|^{2} \\
& \approx \prod_{i=1}^{N} \frac{\min \left(N_{\nu_{i}}, N_{\max }\right)}{N_{\nu_{i}}} .
\end{aligned}
$$

Here, we make several approximations. We assume that only the diagonal terms in the sum $\sum_{P, P^{\prime}}$ survive (i.e., those terms with $P=P^{\prime}$ ). This necessarily would happen if $N_{\max }>N_{\nu_{i}}$ for all $\nu_{i}$, but because we are restricting the sum, this is merely an approximation. However, it should be a good one given that the matrix elements are bounded and oscillating in sign. Finally, we approximate the sum $\sum_{|n| \leq N_{\max }}\left|c_{\nu, n}\right|^{2}=\left[\min \left(N_{\nu_{i}}, N_{\max }\right)\right] / N_{\nu_{i}}$. This is reasonable given that the coefficients $c_{\nu, n}$ are oscillating with roughly uniform amplitude in the range $n \in\left(-N_{\nu}, N_{\nu}\right)$.

We now need to consider the overlaps of $\left|\psi_{\alpha \text {,cos }}\right\rangle$ with the pre-quench ground state, i.e., $c_{\alpha}=\left\langle\nu_{1}, \ldots, \nu_{N} \mid \chi_{1}, \ldots, \chi_{N}\right\rangle$. As before, the square of this overlap can be written as 


$$
\begin{aligned}
\left|\left\langle\nu_{1}, \ldots, \nu_{N} \mid \chi_{1}, \ldots, \chi_{N}\right\rangle\right|^{2}= & \sum_{P, P^{\prime}} \operatorname{sign}(P) \operatorname{sign}\left(P^{\prime}\right) \\
& \times \prod_{i=1}^{N}\left\langle\nu_{P_{i}} \mid \chi_{i}\right\rangle\left\langle\chi_{i} \mid \nu_{P_{i}^{\prime}}\right\rangle \\
& =\sum_{P} \prod_{i=1}^{N}\left|\left\langle\nu_{P_{i}} \mid \chi_{i}\right\rangle\right|^{2},
\end{aligned}
$$

where we suppose that this sum is again dominated by its diagonal terms. This is justified (weakly) in that we will be performing partial sums over the $\nu_{i}$ 's that will (by orthogonality) provide a partial projection of the off-diagonal $\left(P \neq P^{\prime}\right)$ terms. We can approximate the single-particle overlaps $|\langle\nu \mid \chi\rangle|^{2}$ as follows:

$$
|\langle\nu \mid \chi\rangle|^{2} \sim \Theta\left(N_{A}-\frac{\nu n_{\cos }}{2}\right) \frac{1}{2 N_{A}} .
$$

Here, we are using the fact that Mathieu functions with $|\nu| \leq 2 N_{A} / n_{\cos }$ (there are $2 N_{A}$ of them in total) will have an appreciable overlap with the Hermite function $\chi$ as such Mathieu functions have Fourier transforms that are spread over a wide range of wave vectors with approximately equal weight. Those Mathieu functions with $|\nu|>2 N_{A} / n_{\text {cos }}$ are approximately plane waves with a large wave vector and, as such, will have an exponentially small overlap with the Hermite functions, $\chi$. We thus approximate these overlaps as zero.

With this result, we can write down an expression for $X_{\text {elim }}=W_{\text {elim }}^{1 / 2}$ :

$$
\begin{aligned}
X_{\text {elim }} & =\sum_{\substack{2 N_{A} / n_{\cos } \leq \Sigma_{1}<<<\nu_{N} \leq 2 N_{A} / n_{\cos } \\
-N_{\max } \leq I_{1}<\cdots}}\left|c_{\alpha}\right|^{2} \mid c_{N} \leq N_{\max } \\
& =\prod_{i=1}^{N} \frac{1}{2 N_{A}} \sum_{\substack{I_{i} \\
\left|\nu_{i}\right| \leq \sum_{\cos }}} \frac{\min \left(N_{\nu_{i}}, N_{\max }\right)}{N_{\nu_{i}}} .
\end{aligned}
$$

Before we can evaluate this expression, we need an expression for $N_{\nu}$. With trial and error, we find such an expression to be

$$
N_{\nu}=a+b \sqrt{\frac{\nu n_{\mathrm{cos}}}{2}} N_{A}^{\beta},
$$

with $a \approx 18, b \approx 1.2$, and $\beta \approx 1 / 2$. This expression is approximately independent of system size $L$ and $n_{\text {cos }}$. We can then finish the evaluation of $X_{\text {elim }}$ with the result

$X_{\text {elim }}=\left[\frac{N_{\max }}{N_{A}} \frac{2}{b}\left(1-\frac{a}{b N_{A}} \log \left(1+\frac{b N_{a}}{a}\right)\right)\right]^{N}$.

We see then that unless $N_{\max }$ (and so $N_{Q}=N_{\max }+2$ ) is approximately equal to the number of Mathieu functions that have appreciable spread in Fourier space, $N_{A}$, the fluctuations of $\mathcal{Q}(t)$ that are eliminated are a small fraction of the whole.

\section{APPENDIX C: DEVELOPMENT OF A MAZUR-LIKE INEQUALITY FOR $\mathcal{Q}$}

In this section, we develop a Mazur bound arising from the existence of the effective charges $\mathcal{Q}$ 's on the correlation function $\chi_{k}$, involving an operator $M_{k}$ defined by

$$
\begin{aligned}
\chi_{k}=\lim _{T \rightarrow \infty}\left[\frac { 1 } { T ^ { 2 } } \int _ { 0 } ^ { T } d t d t _ { 0 } \left(\left\langle i\left|M_{k}\left(t+t_{0}\right) M_{k}\left(t_{0}\right)\right| i\right\rangle\right.\right. \\
\left.\left.-\left\langle M_{k}\right\rangle_{\mathrm{DE}}^{2}\right)\right]^{1 / 2} /\left\langle M_{k}\right\rangle_{\mathrm{DE}} ; \\
\left\langle M_{k}\right\rangle_{\mathrm{DE}}=\lim _{T \rightarrow \infty} \frac{1}{T} \int_{0}^{T}\left\langle i\left|M_{k}(t)\right| i\right\rangle .
\end{aligned}
$$

We suppose that the initial condition state $|i\rangle=\left|\psi_{\mathrm{GS} \text {,para }}\right\rangle$ is a superposition of post-quench eigenstates whose energies all fall below a cutoff $\Lambda$.

Now, our basic goal is to show that the existence of the effective charges $\mathcal{Q}$ 's places a lower bound on $\chi_{k}$. The $\mathcal{Q}$ 's that we have constructed take the form

$$
\left|\left\langle j|\mathcal{Q}| j^{\prime}\right\rangle\right|= \begin{cases}<\delta & \text { for } E_{j}, E_{j^{\prime}} \leq \Lambda \\ =\mathcal{O}(1) & \text { for } j=j^{\prime} \\ =\mathcal{O}(1) & \text { for } E_{j} \quad \text { or } \quad E_{j^{\prime}}>\Lambda\end{cases}
$$

where $\delta$ is a dimensionless number.

To demonstrate how $\mathcal{Q}$ controls the time evolution of $M_{k}$, we expand $M_{k}$ as follows:

$$
M_{k}=\alpha_{k} \mathcal{Q}+\sum_{l} \alpha_{k l} \tilde{Q}_{l}+M_{k}{ }^{\prime} .
$$

Here, $\tilde{Q}_{l}$ is some set of operators that are completely diagonal in the post-quench eigenbasis, and $M_{k}^{\prime}$ is a completely off-diagonal operator (in the same eigenbasis). $\tilde{Q}_{l}$ (not to be mistaken for the Lieb-Liniger charges) are such that they are orthogonal both to one another and to $\mathcal{Q}$ :

$$
\begin{aligned}
\left\langle\tilde{Q}_{l} \tilde{Q}_{l^{\prime}}\right\rangle & =\delta_{l l^{\prime}}\left\langle\tilde{Q}_{l}^{2}\right\rangle ; \\
\left\langle\tilde{Q}_{l} \mathcal{Q}\right\rangle & =0 .
\end{aligned}
$$

Because $\mathcal{Q}$ is only (approximately diagonal) on the lowenergy Hilbert space, we divide it into two pieces: one diagonal and one wholly nondiagonal:

$$
\mathcal{Q}=\mathcal{Q}_{\text {diag }}+\mathcal{Q}_{\text {nondiag }} .
$$

With this representation in hand, we now return to $\chi_{k}$. We begin evaluating it by inserting a resolution of the identity between the two fields. We assume the spectrum is nondegenerate: 


$$
\begin{aligned}
\chi_{k}= & \frac{1}{T^{2}} \int_{0}^{T} d t d t_{0} \sum_{j j^{\prime} j^{\prime \prime}} c_{j}^{*} c_{j^{\prime \prime}}\left\langle j\left|M_{k}(0)\right| j^{\prime}\right\rangle \\
& \times\left\langle j^{\prime}\left|M_{k}(0)\right| j^{\prime \prime}\right\rangle e^{i\left(t+t_{0}\right)\left(E_{j}-E_{j^{\prime}}\right)+i t_{0}\left(E_{j^{\prime}}-E_{j^{\prime \prime}}\right)} \\
= & \sum_{j}\left|c_{j}\right|^{2}\left|\left\langle j\left|M_{k}(0)\right| j\right\rangle\right|^{2} .
\end{aligned}
$$

In this form, we see that the off-diagonal parts of $M_{k}$ have been projected away:

$$
\begin{array}{r}
\chi_{k}=\sum_{j}\left|c_{j}\right|^{2}\left|\left\langle j\left|\alpha_{k} \mathcal{Q}+\sum_{l} \alpha_{k l} \tilde{Q}_{l}\right| j\right\rangle\right|^{2} \\
=\sum_{j}\left|c_{j}\right|^{2}\left[\alpha_{k}^{2}\left|\left\langle j\left|\mathcal{Q}_{\text {diag }}\right| j\right\rangle\right|^{2}\right. \\
\left.+\sum_{l} \alpha_{k l}^{2}\left|\left\langle j\left|\tilde{Q}_{l}\right| j\right\rangle\right|^{2}\right],
\end{array}
$$

where in the second line we have used the orthogonality of $\mathcal{Q}$ and the $\tilde{Q}_{l}$ 's with one another. As each term in the above is non-negative, we have the inequality

$$
\chi_{k} \geq \alpha_{k}^{2}\left\langle\mathcal{Q}_{\text {diag }}^{2}\right\rangle .
$$

However, for this to be a meaningful inequality, we must show that $\alpha_{k}$ is finite.

To compute $\alpha_{k}$, we consider the projection of $M_{k}$ against $\mathcal{Q}_{\text {diag }}:$

$$
\begin{aligned}
\left\langle M_{k} \mathcal{Q}_{\text {diag }}\right\rangle & =\alpha_{k}\left\langle\mathcal{Q} \mathcal{Q}_{\text {diag }}\right\rangle+\sum_{l} \alpha_{k l}\left\langle\tilde{Q}_{l} \mathcal{Q}_{\text {diag }}\right\rangle+\left\langle M_{k}{ }^{\prime} \mathcal{Q}_{\text {diag }}\right\rangle \\
& =\alpha_{k}\left\langle\mathcal{Q}_{\text {diag }}^{2}\right\rangle,
\end{aligned}
$$

where in the last line we have used the diagonality of $\mathcal{Q}_{\text {diag }}$ and its orthogonality with the other charges, $\tilde{Q}_{l}$. Thus, $\alpha_{k}$ equals

$$
\alpha_{k}=\frac{\left\langle M_{k} \mathcal{Q}_{\text {diag }}\right\rangle}{\left\langle\mathcal{Q}_{\text {diag }}^{2}\right\rangle} .
$$

By inserting a resolution of the identity between the fields and taking the action of $\mathcal{Q}_{\text {diag }}$ on the post-quench eigenbasis to be

$$
\mathcal{Q}_{\text {diag }}|j\rangle=\mathcal{Q}_{j}|j\rangle,
$$

the above simplifies to

$$
\alpha_{k}=\frac{\sum_{j}\left|c_{j}\right|^{2} \mathcal{Q}_{j}\left\langle j\left|M_{k}\right| j\right\rangle}{\sum_{j}\left|c_{j}\right|^{2} \mathcal{Q}_{j}^{2}},
$$

while the lower bound on $\chi_{k}$ becomes

$$
\chi_{k} \geq \frac{\left(\sum_{j}\left|c_{j}\right|^{2} \mathcal{Q}_{j}\left\langle j\left|M_{k}\right| j\right\rangle\right)^{2}}{\sum_{j}\left|c_{j}\right|^{2} \mathcal{Q}_{j}^{2}} .
$$

[1] A. N. Kolmogorov, On Conservation of Conditionally Periodic Motions for a Small Change in Hamilton's Function, Dokl. Akad. Nauk SSSR 98, 527 (1954).

[2] D. Fioretto and G. Mussardo, Quantum Quenches in Integrable Field Theories, New J. Phys. 12, 055015 (2010).

[3] J.-S. Caux and F.H. L. Essler, Time Evolution of Local Observables After Quenching to an Integrable Model, Phys. Rev. Lett. 110, 257203 (2013).

[4] M. Rigol, V. Dunjko, and M. Olshanii, Thermalization and Its Mechanism for Generic Isolated Quantum Systems, Nature (London) 452, 854 (2008).

[5] M. Rigol, V. Dunjko, V. Yurovsky, and M. Olshanii, Relaxation in a Completely Integrable Many-Body Quantum System: An Ab Initio Study of the Dynamics of the Highly Excited States of $1 D$ Lattice Hard-Core Bosons, Phys. Rev. Lett. 98, 050405 (2007).

[6] M. A. Cazalilla, Effect of Suddenly Turning on Interactions in the Luttinger Model, Phys. Rev. Lett. 97, 156403 (2006).

[7] A. Iucci and M. A. Cazalilla, Quantum Quench Dynamics of the Sine-Gordon Model in Some Solvable Limits, New J. Phys. 12, 055019 (2010).

[8] J.-S. Caux and R. M. Konik, Constructing the Generalized Gibbs Ensemble After a Quantum Quench, Phys. Rev. Lett. 109, 175301 (2012).

[9] J. De Nardis, B. Wouters, M. Brockmann, and J.-S. Caux, Solution for an Interaction Quench in the Lieb-Liniger Bose Gas, Phys. Rev. A 89, 033601 (2014).

[10] M. Kormos, A. Shashi, Y.-Z. Chou, J.-S. Caux, and A. Imambekov, Interaction Quenches in the One-Dimensional Bose Gas, Phys. Rev. B 88, 205131 (2013).

[11] D. Iyer and N. Andrei, Quench Dynamics of the Interacting Bose Gas in One Dimension, Phys. Rev. Lett. 109, 115304 (2012).

[12] H. Castella, X. Zotos, and P. Prelovšek, Integrability and Ideal Conductance at Finite Temperatures, Phys. Rev. Lett. 74, 972 (1995).

[13] J. Sirker, R. G. Pereira, and I. Affleck, Diffusion and Ballistic Transport in One-Dimensional Quantum Systems, Phys. Rev. Lett. 103, 216602 (2009).

[14] D. Rossini, A. Silva, G. Mussardo, and G. E. Santoro, Effective Thermal Dynamics Following a Quantum Quench in a Spin Chain, Phys. Rev. Lett. 102, 127204 (2009).

[15] T. Prosen, Open XXZ Spin Chain: Nonequilibrium Steady State and a Strict Bound on Ballistic Transport, Phys. Rev. Lett. 106, 217206 (2011).

[16] P. Calabrese, F. H. L. Essler, and M. Fagotti, Quantum Quench in the Transverse-Field Ising Chain, Phys. Rev. Lett. 106, 227203 (2011).

[17] P. Calabrese, F. H. L. Essler, and M. Fagotti, Quantum Quench in the Transverse Field Ising Chain I: Time Evolution of Order Parameter Correlators, J. Stat. Mech. (2012) P07016.

[18] P. Calabrese, F. H. L. Essler, and M. Fagotti, Quantum Quench in the Transverse Field Ising Chain II: Stationary State Properties, J. Stat. Mech. (2012) P07022.

[19] M. Collura, S. Sotiriadis, and P. Calabrese, Equilibration of a Tonks-Girardeau Gas Following a Trap Release, Phys. Rev. Lett. 110, 245301 (2013).

[20] M. Collura, S. Sotiriadis, and P. Calabrese, Quench Dynamics of a Tonks-Girardeau Gas Released from a Harmonic Trap, J. Stat. Mech. (2013) P09025. 
[21] B. Wouters, M. Brockmann, J. De Nardis, D. Fioretto, M. Rigol, and J.-S. Caux, Quenching the Anisotropic Heisenberg Chain: Exact Solution and Generalized Gibbs Ensemble, Phys. Rev. Lett. 113, 117202 (2014).

[22] B. Pozsgay, M. Mestyán, M. A. Werner, M. Kormos, G. Zaránd, and G. Takács, Correlations After Quantum Quenches in the XXZ Spin Chain: Failure of the Generalized Gibbs Ensemble, Phys. Rev. Lett. 113, 117203 (2014).

[23] G. Goldstein and N. Andrei, Failure of the GGE Hypothesis for Integrable Models with Bound States, Phys. Rev. A 90, 043625 (2014).

[24] M. Mestyan, B. Pozsgay, G. Takacs, and M. A. Werner, Quenching the XXZ Spin Chain: Quench Action Approach Versus Generalized Gibbs Ensemble, arXiv:1412.4787.

[25] B. Pozsgay, Failure of the Generalized Eigenstate Thermalization Hypothesis in Integrable Models with Multiple Particle Species, arXiv:1406.4613.

[26] S. Sotiriadis, G. Takacs, and G. Mussardo, Boundary State in an Integrable Quantum Field Theory out of Equilibrium, Phys. Lett. B 734, 52(2014).

[27] F. H. L. Essler, G. Mussardo, and M. Panfil, Generalized Gibbs Ensembles for Quantum Field Theories, Phys. Rev. A 91, 051602 (2015).

[28] E. Ilievski, M. Medenjak, and T. Prosen, Quasilocal Conserved Operators in Isotropic Heisenberg Spin 1/2 Chain, Phys. Rev. Lett. 114, 140601 (2015).

[29] E. Ilievski, J. De Nardis, B. Wouters, J.-S. Caux, F. H. L. Essler, and T. Prosen, Complete Generalized Gibbs Ensemble in an Interacting Theory, arXiv:1507.02993.

[30] T. Kinoshita, T. Wenger, and D.S. Weiss, A Quantum Newton's Cradle, Nature (London) 440, 900 (2006).

[31] M. Gring, M. Kuhnert, T. Langen, T. Kitagawa, B. Rauer, M. Schreitl, I. Mazets, D. Adu Smith, E. Demler, and J. Schmiedmayer, Relaxation and Pre-thermalization in an Isolated Quantum System, Science 337, 1318 (2012).

[32] D. Adu Smith, M. Gring, T. Langen, M. Kuhnert, B. Rauer, R. Geiger, T. Kitagawa, I. Mazets, E. Demler, and J. Schmiedmayer, Prethermalization Revealed by the Relaxation Dynamics of Full Distribution Functions, New J. Phys. 15, 075011 (2013).

[33] F. Meinert, M. J. Mark, E. Kirilov, K. Lauber, P. Weinmann, M. Gröbner, and H.-C. Nägerl, Interaction-Induced Quantum Phase Revivals and Evidence for the Transition to the Quantum Chaotic Regime in 1D Atomic Bloch Oscillations, Phys. Rev. Lett. 112, 193003 (2014).

[34] D. Basko, I. Aleiner, and B. Altshuler, Metal-Insulator Transition in a Weakly Interacting Many-Electron System with Localized Single-Particle States, Ann. Phys. (Amsterdam) 321, 1126 (2006).

[35] V. Oganesyan and D. A. Huse, Localization of Interacting Fermions at High Temperature, Phys. Rev. B 75, 155111 (2007).

[36] M. Serbyn, Z. Papić, and D. A. Abanin, Local Conservation Laws and the Structure of the Many-Body Localized States, Phys. Rev. Lett. 111, 127201 (2013).

[37] J. Z. Imbrie, On Many-Body Localization for Quantum Spin Chains, arXiv:1403.7837.

[38] M. V. Berry and M. Tabor, Level Clustering in the Regular Spectrum, Proc. R. Soc. A 356, 375 (1977).
[39] G. P. Brandino, R. M. Konik, and G. Mussardo, Energy Level Distribution of Perturbed Conformal Field Theories, J. Stat. Mech. (2010) P07013.

[40] M. Rigol, Breakdown of Thermalization in Finite OneDimensional Systems, Phys. Rev. Lett. 103, 100403 (2009).

[41] M. Rigol, Quantum Quenches and Thermalization in OneDimensional Fermionic Systems, Phys. Rev. A 80, 053607 (2009).

[42] I. C. Percival, Regular and Irregular Spectra, J. Phys. B 6, L229 (1973).

[43] M. V. Berry, Regular and Irregular Semiclassical Wavefunctions, J. Phys. A 10, 2083 (1977).

[44] A. Voros, Stochastic Behavior in Classical and Quantum Hamiltonian Systems (Springer, Berlin, 1979).

[45] J. Berges, S. Borsányi, and C. Wetterich, Prethermalization, Phys. Rev. Lett. 93, 142002 (2004).

[46] M. Moeckel and S. Kehrein, Interaction Quench in the Hubbard Model, Phys. Rev. Lett. 100, 175702 (2008).

[47] M. Moeckel and S. Kehrein, Crossover from Adiabatic to Sudden Interaction Quenches in the Hubbard Model: Prethermalization and Nonequilibrium Dynamics, New J. Phys. 12, 055016 (2010).

[48] M. Kollar, F. A. Wolf, and M. Eckstein, Generalized Gibbs Ensemble Prediction of Prethermalization Plateaus and Their Relation to Nonthermal Steady States in Integrable Systems, Phys. Rev. B 84, 054304 (2011).

[49] F. H. L. Essler, S. Kehrein, S. R. Manmana, and N. J. Robinson, Quench Dynamics in a Model with Tuneable Integrability Breaking, Phys. Rev. B 89, 165104 (2014).

[50] B. Bertini, F. H. L. Essler, S. Groha, and N. J. Robinson, Prethermalization and Thermalization in Models with Weak Integrability Breaking, arXiv:1506.02994.

[51] M. Fagotti, On Conservation Laws, Relaxation and Pre-relaxation After a Quantum Quench, J. Stat. Mech. (2014) P03016.

[52] B. Bertini and M. Fagotti, Pre-relaxation in Weakly Interacting Models, J. Stat. Mech. (2015) P07012.

[53] M. Fagotti and M. Collura, Universal Prethermalization Dynamics of Entanglement Entropies After a Global Quench, arXiv:1507.02678.

[54] N. Nekhoroshev, Behavior of Hamiltonian Systems Close to Integrable, Funct. Anal. Appl. 5, 338 (1971).

[55] J. Pöschel, On Nekhoroshev's Estimate for Quasi-convex Hamiltonians, Math. Z. 213, 187 (1993).

[56] E. H. Lieb and W. Liniger, Exact Analysis of an Interacting Bose Gas. I. The General Solution and the Ground State, Phys. Rev. 130, 1605 (1963).

[57] M. A. Cazalilla, R. Citro, T. Giamarchi, E. Orignac, and M. Rigol, One Dimensional Bosons: From Condensed Matter Systems to Ultracold Gases, Rev. Mod. Phys. 83, 1405 (2011).

[58] R. M. Konik and Y. Adamov, Numerical Renormalization Group for Continuum One-Dimensional Systems, Phys. Rev. Lett. 98, 147205 (2007).

[59] R. M. Konik, Exciton Hierarchies in Gapped Carbon Nanotubes, Phys. Rev. Lett. 106, 136805 (2011).

[60] J.-S. Caux, Correlation Functions of Integrable Models: A Description of the ABACUS Algorithm, J. Math. Phys. (N.Y.) 50, 095214 (2009). 
[61] J.-S. Caux, Correlation Functions of Integrable Models: A Description of the ABACUS Algorithm, J. Math. Phys. (N.Y.) 50, 095214 (2009).

[62] V.P. Yurov and A. B. Zamolodchikov, Truncated Conformal Space Approach to Scaling Lee-Yang Model, Int. J. Mod. Phys. A 05, 3221 (1990).

[63] V.P. Yurov and Al. B. Zamolodchikov, Truncated Fermionic Space Approach to the Critical 2-D Ising Model with Magnetic Field, Int. J. Mod. Phys. A 06, 4557 (1991).

[64] B. Davies and V. E. Korepin, Higher Conservation Laws for the Quantum Non-linear Schroedinger Equation, arXiv: 1109.6604.

[65] We have not made a systematic study of how the coefficients $a_{i}$ forming the linear combination depend on $N_{Q}$, the number of charges forming the effective charge. Ideally, one would want the $a_{i}$ to be more heavily weighted for $i$ small so as to build a more local effective charge. Our minimization procedure by which the $a_{i}$ 's are constructed does not necessarily ensure this. However, in a future work, we plan to look at a weighted minimization scheme for the fixing of the $a_{i}$ 's that take into account a desire for locality. Nonetheless, we do point out that the charges we construct here, even without controlling explicitly for locality, do control long time dynamics as evidenced in Sec. IV A.

[66] V. A. Yurovksy and M. Olshanii, Memory of the Initial Conditions in an Incompletely Chaotic Quantum System: Universal Predictions with Application to Cold Atoms, Phys. Rev. Lett. 106, 025303 (2011).

[67] M. Olshanii, K. Jacobs, M. Rigol, V. Dunjko, H. Kennard, and V. A. Yurovsky, An Exactly Solvable Model for the Integrability-Chaos Transition in Rough Quantum Billiards, Nat. Commun. 3, 641 (2012).

[68] Even though the parabolic potential mixes states with different momenta more strongly than a cosine potential, it does not strongly connect the low- and high-energy parts of the unperturbed Lieb-Liniger eigenspace.

[69] L. F. Santos and M. Rigol, Onset of Quantum Chaos in One-Dimensional Bosonic and Fermionic Systems and Its Relation to Thermalization, Phys. Rev. E 81, 036206 (2010).

[70] L. F. Santos and M. Rigol, Localization and the Effects of Symmetries in the Thermalization Properties of $1 D$ Quantum Systems, Phys. Rev. E 82, 031130 (2010).

[71] P. Mazur, Non-ergodicity of Phase Functions in Certain Systems, Physica (Amsterdam) 43, 533 (1969).

[72] M. Suzuki, Ergodicity, Constants of Motion, and Bounds for Susceptibilities, Physica (Amsterdam) 51, 277 (1971).

[73] See also M. Mierzejewski, T. Prosen, and P. Prelovsek, Approximate Conservation Laws in Perturbed Integrable Lattice Models, arXiv:1508.06385, where approximate conserved charges in models with weak integrability breaking are deployed in the context of Mazur's inequality.
[74] M. Olshanii, Geometry of Quantum Observables and Thermodynamics of Small Systems, Phys. Rev. Lett. 114, 060401 (2015).

[75] K. Wilson, The Renormalization Group: Critical Phenomena and the Kondo Problem, Rev. Mod. Phys. 47, 773 (1975).

[76] N. Nekhoroshev, Calculation of Scalar Products of Wave Functions and Form-Factors in the Framework of the Algebraic Bethe Ansatz, Theor. Math. Phys. 79, 502 (1989).

[77] N. A. Slavnov, Nonequal-Time Current Correlation Function in a One-Dimensional Bose Gas, Theor. Math. Phys. 82, 273 (1990).

[78] J.-S. Caux, P. Calabrese, and N. Slavnov, One-Particle Dynamical Correlations in the One-Dimensional Bose Gas, J. Stat. Mech. (2007) P01008.

[79] J.-S. Caux and P. Calabrese, Correlation Functions of the One-Dimensional Attractive Bose Gas, Phys. Rev. A 74, 031605 (2006).

[80] S. R. White, Density Matrix Formulation for Quantum Renormalization Groups, Phys. Rev. Lett. 69, 2863 (1992).

[81] S. R. White, Density-Matrix Algorithms for Quantum Renormalization Groups, Phys. Rev. B 48, 10345 (1993).

[82] M. Khodas, M. Pustilnik, A. Kamenev, and L. I. Glazman, Dynamics of Excitations in a One-Dimensional Bose Liquid, Phys. Rev. Lett. 99, 110405 (2007).

[83] L. Tonks, The Complete Equation of State of One, Two and Three-Dimensional Gases of Hard Elastic Spheres, Phys. Rev. 50, 955 (1936).

[84] M.D. Girardeau, Relationship between Systems of Impenetrable Bosons and Fermions in One Dimension, J. Math. Phys. (N.Y.) 1, 516 (1960).

[85] Even in the Tonk-Girardeau limit, the wave functions of the bosonic and of the fermionic system are not the same, but they are connected by

$$
\psi_{B}\left(x_{1}, \ldots, x_{N}\right)=\left|\psi_{F}\left(x_{1}, \ldots, x_{N}\right)\right| .
$$

The two wave functions coincide in one of the sectors, say, $x_{1}<x_{2}, \ldots,<x_{N}$, but differ by their symmetry with respect to the permutation of particles coordinates. While some observables are sensitive to this difference (for example, the momentum distribution), the density operator (which we will be interested in later for computing the profile of the gas in the potential) does not permute particles; its matrix elements between any two many-body eigenstates of $H_{B}$ are identical to those evaluated with the corresponding dual eigenstates of $H_{F}$.

[86] Handbook of Mathematical Functions with Formulas, Graphs, and Mathematical Tables, National Bureau of Standards, Applied Mathematics Series No. 55, 10th ed., edited by M. Abramowitz and I. Stegun (U.S. Govt. Printing Office, Washington, D.C., 1972), p. 721. 